\title{
Approximation in ergodic theory, Borel, and Cantor dynamics
}

\author{
S. Bezuglyi, J. Kwiatkowski, and K. Medynets
}

\begin{abstract}
This survey is focused on the results related to topologies on the groups of transformations in ergodic theory, Borel, and Cantor dynamics. Various topological properties (density, connectedness, genericity) of these groups and their subsets (subgroups) are studied.
\end{abstract}

In this paper, we intend to present a unified approach to the study of topological properties of transformation groups arisen in ergodic theory, Borel, and Cantor dynamics. We consider here the group $\operatorname{Aut}(X, \mathcal{B}, \mu)$ (and $\left.\operatorname{Aut}_{0}(X, \mathcal{B}, \mu)\right)$ of all non-singular (measure preserving, resp.) automorphisms of a standard measure space, the group $A u t(X, \mathcal{B})$ of all Borel automorphisms of a standard Borel space, and the group Homeo $(\Omega)$ of all homeomorphisms of a Cantor set $\Omega$. The basic technique in the study of transformation groups acting on an underlying space is to introduce various topologies into these groups which make them topological groups and investigate topological properties of the groups and their subsets (subgroups).

The study of topologies on the group of transformations of a space has a long history. The first significant results in this area are the classical results of Oxtoby and Ulam on the typical dynamical behavior of homeomorphisms which preserve a measure $\mathrm{O}-\mathrm{U}$. This circle of problems has attracted attention in various areas of dynamical systems, notably, in measurable and topological dynamics, where it is important for many applications to understand what kind of transformations is typical for certain dynamics. Of course, this problem assumes that a topology is defined on the group of all transformations.

The topologies which are usually considered on the groups $\operatorname{Aut}(X, \mathcal{B}, \mu)$ and $A u t_{0}(X, \mathcal{B}, \mu)$ were defined 60 years ago in the pioneering paper by Halmos Hal 1 where he called them the uniform and weak topologies. The use of these topologies turned out to be very fruitful and led to many outstanding results in ergodic theory. For instance, the best known theorems concerning ergodic, mixing, and weakly mixing automorphisms of a measure space were obtained by P. Halmos and

1991 Mathematics Subject Classification. Primary 37A40, 37B05, 03066.

Key words and phrases. automorphism, measure space, standard Borel space, Cantor set, homeomorphism.

S. Bezuglyi was supported in part by CRDF grant UM1-2546-KH-03.

J. Kwiatkowski was supported in part by KBN grant 1P 03A 03826.

(C)0000 (copyright holder) 
V.A. Rokhlin (see [Hal 1, Hal 2, Ro 1], Ro 2]). Many further statements on approximation of automorphisms of a measure space can be found in the book [C-F-S] and in a great number of research and expository papers written in last decades (see, e.g., the references to this survey).

Motivated by ideas used in ergodic theory, we define several topologies on the groups $A u t(X, \mathcal{B})$ and $\operatorname{Homeo}(\Omega)$ which are similar to the weak and uniform topologies. They were first introduced in the context of Cantor dynamics in B-K 1 and B-K 2] where we marked that many results in Cantor dynamics have their counterparts ergodic theory. Then, we used these topologies to the study of the groups $\operatorname{Aut}(X, \mathcal{B})$ and $\mathrm{Homeo}_{(\Omega)}$ in the series of papers [B-D-K 1], [B-D-K 2], [B-D-M], B-M]. To the best our knowledge, topologies on the group $\operatorname{Aut}(X, \mathcal{B})$ had not been considered before. On the other hand, there exist a few useful topologies on the group of homeomorphisms of a topological space $K$. The most known of them is the topology of uniform convergence on $H_{o m e o}(K)$ (or the compact-open topology). Many remarkable results about topological properties of Homeo(K) were obtained for a connected compact metric space $K$ (see, e.g. Alp-Pr 2 and the references there).

In our study of the groups $\operatorname{Aut}(X, \mathcal{B})$ and $\operatorname{Homeo}(\Omega)$, we noticed that most of topological properties known for $\operatorname{Aut}(X, \mathcal{B}, \mu)$ hold also for Borel and Cantor dynamics. Based on this observation, we have tried to systematize the known results for measurable, Borel, and Cantor dynamics to emphasize some common features and point out the existing distinguishes. In the article, we consider the global topological structure of these large groups. We also study some classes (subsets, subgroups) of transformations which are naturally defined, for instance, periodic, aperiodic, minimal, odometers, etc. The closures of these classes are found in the topologies we have defined. We consider here the problems and results which are parallel in ergodic theory, Borel, and Cantor dynamics. It will be seen below that the groups we study have a lot of similar topological properties. This observation is a justification for studying topologies on the groups of transformations in an unified manner.

The paper is organized as follows. In the first section, we collect main definitions and notions used in ergodic theory, Borel, and Cantor dynamics. Our goal is twofold: we fix the notation used in the paper and try to make the paper self-contained (this may be useful for non-specialists). Section 2 contains the well known results on applications of the weak and uniform topologies in ergodic theory. We do not intend to give a comprehensive description of all results related to topological properties of $\operatorname{Aut}(X, \mathcal{B}, \mu)$ and $\operatorname{Aut}_{0}(X, \mathcal{B}, \mu)$. They are too numerous to list here. Our choice is explained by interest and knowledge, and, all above, by our study of topologies in the context of Borel and Cantor dynamics. The next two sections contain the results on topologies on the groups $\operatorname{Aut}(X, \mathcal{B})$ and Homeo $(\Omega)$. We define two topologies $\tau$ and $p$ which are analogous to the uniform and weak topologies in measurable dynamics and formulate many results on topological properties of these groups and various subsets and subgroups. In particular, we consider periodic, aperiodic, minimal transformations, odometers etc. In the last section, we give a comparison of results obtained in measurable, Borel, and Cantor dynamics.

While preparing this survey, we improved several results from our previous works and included them in the paper with sketches of the proofs. We also used the nice written papers [Ch-Pr, Alp-Pr 2 where one can find many results on 
topological properties of the group of automorphisms of a measure space and the group of measure-preserving homeomorphisms of a compact connected manifold.

In this survey, we do not discuss many remarkable results which have been published during last decade in Borel and Cantor dynamics. In this connection, we refer to G-P-S 1], G-P-S 3], Hj], J-Ke-L, Ke-Mil where further references can be found.

\section{Preliminaries}

In this section, we recall main notions and definitions from ergodic theory (called also measurable dynamics), Borel, and Cantor dynamics which are used in the paper. We will keep the notation from this section throughout the paper.

1.1. Borel dynamics. Let $X$ be a topological space. The class $\mathcal{B}(X)=\mathcal{B}$ of Borel sets of $X$ is the $\sigma$-algebra generated by open sets. We call $(X, \mathcal{B})$ the Borel space. A separable complete metric space $Y$ is called a Polish space. Let $\mathcal{C}$ be the $\sigma$-algebra of Borel subsets of $Y$. A Borel space $(X, \mathcal{B})$ is called a standard Borel space if it is isomorphic to a Polish space $(Y, \mathcal{C})$. Any two standard Borel spaces are isomorphic.

Let $\mathcal{M}_{1}(X)$ denote the set of all Borel probability measures on $(X, \mathcal{B})$. A measure $\mu \in \mathcal{M}_{1}(X)$ is called non-atomic (or continuous) if $\mu(\{x\})=0 \forall x \in X$. The measure, supported by a point $x \in X$, is denoted by $\delta_{x}$. Two measures $\mu$ and $\nu$ are called equivalent (in symbols, $\mu \sim \nu$ ) if they have the same collection of subsets of measure zero.

A one-to-one Borel map $T$ of $X$ onto itself is called an automorphism of $(X, \mathcal{B})$. Denote by $\operatorname{Aut}(X, \mathcal{B})$ the group of all Borel automorphisms of $(X, \mathcal{B})$. For $T \in$ $\operatorname{Aut}(X, B)$, the set $\operatorname{supp}(T)=\{x \in X: T x \neq x\}$ is called the support of $T$. Let $C t b l(X)$ denote the set of automorphisms with at most countable support, i.e., $T \in C t b l(X)$ if $|(\operatorname{supp}(T))| \leq \aleph_{0}$. Clearly, $C t b l(X)$ is a normal subgroup of $\operatorname{Aut}(X, B)$. The quotient group $\widehat{\operatorname{Aut}}(X, B)=\operatorname{Aut}(X, B) / C t b l(X)$ is obtained by identifying automorphisms from $\operatorname{Aut}(X, \mathcal{B})$ which differ on an at most countable set.

For $T \in \operatorname{Aut}(X, \mathcal{B})$ and $x \in X$, let $\operatorname{Orb}_{T}(x)=\left\{T^{n} x: n \in \mathbb{Z}\right\}$ denote the $T$-orbit of $x$. A point $x \in X$ is called a periodic point of period $n$ if $T^{n} x=x$ and $T^{i} x \neq x, i=1, \ldots, n-1$. An automorphism $T$ is called pointwise periodic (or periodic) if every point $x \in X$ is periodic for $T$, and $T$ is called aperiodic if it has no periodic points, i.e. every $T$-orbit is infinite. We denote by $\mathcal{P} e r$ and $\mathcal{A} p$ the sets of pointwise periodic and aperiodic automorphisms, respectively.

A Borel automorphism $T \in A u t(X, \mathcal{B})$ is called smooth if there is a Borel subset $A \subset X$ (called wandering) that meets every $T$-orbit exactly ones. We denote the class of smooth automorphisms by $\mathcal{S} m$. By our definition, every pointwise periodic automorphism is smooth.

Given $T \in \operatorname{Aut}(X, \mathcal{B})$, denote by $M_{1}(T)$ the set of $T$-invariant Borel probability measures. In particular, $M_{1}(T)$ may be empty. An automorphism $T$ is called incompressible if $M_{1}(T) \neq \emptyset$ (in symbols, $T \in \mathcal{I} n c$ ).

By definition, the full group of $T \in \operatorname{Aut}(X, \mathcal{B})$ is $[T]=\{S \in \operatorname{Aut}(X, \mathcal{B})$ : $\left.\operatorname{Orb}_{S}(x) \subset \operatorname{Orb}_{T}(x), x \in X\right\}$.

We recall also the definition of odometers. Let $\left\{p_{n}\right\}_{n=0}^{\infty}$ be a sequence of integers such that $p_{n} \geq 2$. Let $\Omega=\prod_{n}\left\{0,1, \ldots, p_{n}-1\right\}$ be endowed with the product topology. Then $\Omega$ is a Cantor set. Let $S: \Omega \rightarrow \Omega$ be defined as follows: $S\left(p_{0}-\right.$ 
$\left.1, p_{1}-1, \ldots\right)=(0,0, \ldots)$, and for any other $x=\left(x_{k}\right) \in X$, find the least $k$ such that $x_{k} \neq p_{k}-1$ and set $S(x)=\left(0,0, \ldots, 0, x_{k}+1, x_{k+1}, x_{k+2}, \ldots\right)$. More general, a Borel automorphism $T$ is called an odometer if it is Borel isomorphic to some $S$. In other words, there exists a sequence of refining partitions of $X$ such that each partition is a $T$-tower and the elements of the partitions generate $\mathcal{B}$. We will denote by $\mathcal{O} d$ the set of odometers.

1.2. Measurable dynamics. A measure space $(X, \mathcal{A}, \mu)$ consists of a set $X$, a $\sigma$-algebra $\mathcal{A}$ of subsets of $X$, and a measure (finite or $\sigma$-finite) $\mu$ on $\mathcal{A}$ with respect to which $\mathcal{A}$ is complete. If $T$ is a one-to-one measurable map of $X$ onto itself, then we can define the measure $\mu \circ T(A):=\mu(T A), A \in \mathcal{A}$. If $\mu \circ T \sim \mu$, then $T$ is called a non-singular automorphism of $(X, \mathcal{A}, \mu)$. If $\mu \circ T=\mu$, then $T$ is called measure-preserving. The group of all non-singular automorphisms is denoted by $\operatorname{Aut}(X, \mathcal{A}, \mu)$, and that of measure-preserving automorphisms is denoted by $\operatorname{Aut}_{0}(X, \mathcal{A}, \mu)$. As usual in ergodic theory, we use the "mod 0 convention", that is all equalities between sets, automorphisms, etc. are to be understood modulo null sets.

We say that $(X, \mathcal{B}, \mu)$ is a standard measure space (or Lebesgue space) if it is isomorphic to $([0, a], \lambda)$ where $a \in[0, \infty]$ and $\lambda$ is the Lebesgue measure. ${ }^{1}$ If $\mu(X)=$ 1 , then $(X, \mathcal{B}, \mu)$ is called a probability standard measure space. To illustrate these definitions, we note that any uncountable Borel subset of a Polish space together with a Borel measure on it is an example of a standard measure space.

An automorphism $T \in \operatorname{Aut}(X, \mathcal{B}, \mu)$ is called ergodic (or $\mu$-ergodic) if for every $T$-invariant set $A \in \mathcal{B}$ one has either $\mu(A)=0$ or $\mu(X \backslash A)=0$. An automorphism $T \in \operatorname{Aut}(X, \mathcal{B}, \mu)$ is called mixing if $\mu\left(T^{-k} A \cap B\right) \rightarrow \mu(A) \mu(B)$ as $k \rightarrow \infty$ for all $A, B \in \mathcal{B}$. $T$ is weakly mixing if $T \times T$ is $\mu \times \mu$-ergodic on $X \times X$. The full group $[T]$ generated by $T \in A u t(X, \mathcal{B}, \mu)$ consists of those automorphisms whose orbits are contained in $T$-orbits. By definition, the normalizer $N[T]$ of $[T]$ is the set $\left\{S \in \operatorname{Aut}(X, \mathcal{B}, \mu): S[T] S^{-1}=[T]\right\}$, and the centralizer $C(T)$ of $T$ is the set $\{S \in \operatorname{Aut}(X, \mathcal{B}, \mu): S T=T S\}$.

Recall that an automorphism $T \in \operatorname{Aut}(X, \mathcal{B}, \mu)$ is called of type $I I$ if there exists a $T$-invariant measure $\nu$ equivalent to $\mu$. If $\nu$ is finite, then $T$ is of type $I I_{1}$; if $\nu$ is infinite then $T$ is of type $I I_{\infty}$. If $T$ does not admit an invariant (finite or infinite) measure equivalent to $\mu$, then $T$ is called of type $I I I$. Automorphisms of type $I I I$ are divided into the subtypes $I I I_{0}, I I I_{\lambda}(0<\lambda<1)$, and $I I I_{1}$ (see Ha-Os for details).

1.3. Topological dynamics. Let $K$ be a compact metric space. Denote by Homeo $(K)$ the group of all homeomorphisms of $K$. We say that $T \in \operatorname{Homeo}(K)$ is topologically transitive if there is $x \in K$ such that $\operatorname{Orb}_{T}(x)$ is dense in $K$. Also, $T$ is called minimal if every $T$-orbit is dense in $K$ or, equivalently, $T$ has no proper closed invariant subsets. It is said that $T \in \operatorname{Homeo}(K)$ is mixing if for every non-empty open subsets $U, V$ there exists $N \in \mathbb{N}$ such that $T^{n} U \cap V \neq \emptyset$ for all $n \geq N$. The sets of all minimal and mixing homeomorphisms will be denoted by $\mathcal{M i n}$ and $\mathcal{M i x}$, respectively. A homeomorphism $T$ of $K$ is called weakly mixing if $T \times T$ is transitive on $K \times K$. Notice that an odometer $S$ is obviously a minimal homeomorphism.

\footnotetext{
${ }^{1}$ In contrast to the original definition, we consider here only non-atomic measures on $X$.
} 
We say that $\Omega$ is a Cantor set if it is homeomorphic to a compact metric space without isolated points whose base consists of clopen sets. All Cantor sets are homeomorphic.

For aperiodic $T \in \operatorname{Homeo}(\Omega)$, define the full group of $T$ as $[T]=\{S \in$ $\operatorname{Homeo}_{(\Omega)}: \operatorname{Orb}_{S}(x) \subset \operatorname{Orb}_{T}(x)$ for all $\left.x \in \Omega\right\}$. Thus, if $S \in[T]$, then $S x=T^{n_{S}(x)} x$ for all $x$, and $x \mapsto n_{S}(x)$ is a Borel map from $\Omega$ to $\mathbb{Z}$. The countable subgroup $[[T]]=\left\{S \in[T]: n_{S}(x)\right.$ is continuous $\}$ is called the topological full group of $T$.

\section{The uniform and weak topologies on $\operatorname{Aut}(X, \mathcal{B}, \mu)$}

In this section, we collect a number of results about topological properties of $\operatorname{Aut}(X, \mathcal{B}, \mu)$ and $\operatorname{Aut}_{0}(X, \mathcal{B}, \mu)$. These results are not new and presented here to emphasize some parallels with results proved for Borel and Cantor dynamics.

2.1. Topological properties of the space $\operatorname{Aut}(X, \mathcal{B}, \mu)$. Let $\operatorname{Aut}(X, \mathcal{B}, \mu)$ be the group of all non-singular measurable automorphisms of a standard measure space $(X, \mathcal{B}, \mu)$ with $\mu(X)=1$. Using the ideas which first appeared in Hal 1, one can introduce two topologies on $\operatorname{Aut}(X, \mathcal{B}, \mu)$ generated by the metrics $d_{u}$ and $d_{w}$. For $S, T \in A u t(X, \mathcal{B}, \mu)$, set

$$
\begin{aligned}
& d_{u}(S, T)=\mu(\{x \in X: S x \neq T x\}), \\
& d_{w}(S, T)=\sum_{n=1}^{\infty} 2^{-n} \mu\left(S A_{n} \Delta T A_{n}\right),
\end{aligned}
$$

where $\left(A_{n}\right)$ is a countable family of measurable subsets generating $\mathcal{B}$.

It is well known that the topologies defined by the metrics (2.1) and (2.2) do not depend on a measure from the class $[\mu]$ of measures equivalent to $\mu$. We will follow the long established tradition in ergodic theory to call them the uniform and weak topologies, respectively. Clearly, the uniform topology is strictly stronger than the weak topology. These topologies on the groups $\operatorname{Aut}(X, \mathcal{B}, \mu)$ and $A u t_{0}(X, \mathcal{B}, \mu)$ have been studied in many papers. We summarize the obtained results on topological and algebraic properties of these groups in the next two theorems.

Theorem 2.1 (uniform topology). (1) The uniform topology on Aut $(X, \mathcal{B}, \mu)$ is equivalent to the topology generated by the metric ${ }^{2}$

$$
d_{u}^{\prime}(S, T)=\sup _{A \in \mathcal{B}} \mu(S A \Delta T A) .
$$

(2) $\left(\operatorname{Aut}(X, \mathcal{B}, \mu), d_{u}\right)$ is a non-separable complete metric space and a topological group.

(3) $\operatorname{Aut}_{0}(X, \mathcal{B}, \mu)$ is closed in $\left(\operatorname{Aut}(X, \mathcal{B}, \mu), d_{u}\right)$;

(4) $\operatorname{Aut}(X, \mathcal{B}, \mu)$ (and $\operatorname{Aut}_{0}(X, \mathcal{B}, \mu)$ ) has no outer group automorphisms and has no closed normal subgroups with respect to the uniform topology.

(5) The topological space $\left(A u t(X, \mathcal{B}, \mu), d_{u}\right)$ is path-connected and simple connected.

(6) The topological space $\operatorname{Aut}_{0}(X, \mathcal{B}, \mu)$ is contractible in the uniform topology.

\footnotetext{
${ }^{2}$ This fact is a justification of the name 'uniform' for this topology.
} 
Some of these results are rather simple exercises which are known for a long time. Statements (1) and (2) were firstly proved in Hal 1 for the group $A_{u} t_{0}(X, \mathcal{B}, \mu)$ and then they were used by many authors in more general settings (see e.g. IT, Ch-Kak, B-Gol, Alp-Pr 2 ). The algebraic and topological structures of $\operatorname{Aut}(X, \mathcal{B}, \mu)$ (and $\operatorname{Aut}_{0}(X, \mathcal{B}, \mu)$ ) were studied in Cha-Fr, B-Gol, E 1], E 2], and some other papers. The fact that the space $\operatorname{Aut}(X, \mathcal{B}, \mu)$ is pathconnected and even simple connected was proved in B-Gol. Notice also that the contractibility of $A_{u} t_{0}(X, \mathcal{B}, \mu)$ was established in Kea.

Let $U_{T}$ be the unitary operator $U_{T}$ acting on $L^{1}(X, \mathcal{B}, \mu)$ as follows:

$$
\left(U_{T} f\right)(x)=f\left(T^{-1} x\right) \sqrt{\frac{d \mu \circ T^{-1}}{d \mu}(x)} .
$$

$U_{T}$ is called the operator associated to $T \in \operatorname{Aut}(X, \mathcal{B}, \mu)$.

The group $\operatorname{Aut}(X, \mathcal{B}, \mu)$ endowed with weak topology has the following topological properties.

Theorem 2.2 (weak topology). (1) $\operatorname{Aut}(X, \mathcal{B}, \mu)$ is a Polish space and a topological group with respect to the weak topology.

(2) A sequence of automorphisms $\left(T_{n}\right)$ converges to $T$ in the weak topology if and only if $\left(U_{T_{n}}\right)$ converges to $U_{T}$ in the strong (weak) operator topology.

(3) The groups $\operatorname{Aut}_{0}(X, \mathcal{B}, \mu)$ and $\operatorname{Aut}(X, \mathcal{B}, \mu)$ are contractible in the weak topology.

(4) $\operatorname{Aut}_{0}(X, \mathcal{B}, \mu)$ is closed in $\operatorname{Aut}(X, \mathcal{B}, \mu)$ with respect to the weak topology.

(5) The set $\bigcup_{\nu \sim \mu} A u t_{0}(X, \mathcal{B}, \nu)$ is a meager subset in $\left(A u t(X, \mathcal{B}, \mu), d_{w}\right)$.

Notice that statement (3) was proved in Kea for the group $A u t_{0}(X, \mathcal{B}, \mu)$ and then later it was generalized to $\operatorname{Aut}(X, \mathcal{B}, \mu)$ in Dan. Statement (5), which asserts, in other words, that the set of automorphisms of type $I I$ is meager in $\operatorname{Aut}(X, \mathcal{B}, \mu)$, was established in [T] (the first example of a non-singular automorphism which does not in $\bigcup_{\nu \sim \mu} A u t_{0}(X, \mathcal{B}, \nu)$ was found by Ornstein). Observe that since $\left(\operatorname{Aut}(X, \mathcal{B}, \mu), d_{w}\right)$ is separable, one can find various classes of automorphisms which are dense in $\operatorname{Aut}(X, \mathcal{B}, \mu)$ in $d_{w}$. For example, assuming $X=[0,1]$, the set of cyclic binary permutations on $[0,1]$ is dense in $d_{w}$. In $\operatorname{Pr} 1$, the following result was proved: The set of pairs of elements from $\operatorname{Aut}_{0}(X, \mathcal{B}, \mu) \times A u t_{0}(X, \mathcal{B}, \mu)$ which generate a dense subgroup of $A_{u} t_{0}(X, \mathcal{B}, \mu)$ form a dense $G_{\delta}$ subset.

2.2. The Rokhlin lemma and Rokhlin property. In Ro 1], the following fundamental result (called lately the Rokhlin lemma) was proved: Let $T$ be an aperiodic finite measure preserving automorphism of $(X, \mathcal{B}, \mu)$. Then given $n \in \mathbb{N}$ and $\varepsilon>0$, there exists a set $A \in \mathcal{B}$ such that $A \cap T^{i} A=\emptyset, i=1, \ldots, n-1$ and

$$
\mu\left(X \backslash \bigcup_{i=0}^{\infty} T^{i} A\right)<\varepsilon
$$

Based on this result, Rokhlin [Ro 1] showed that the set $\mathcal{P e r}$ of periodic automorphisms is dense in $A_{u} t_{0}(X, \mathcal{B}, \mu)$ with respect to the uniform topology: For $T \in \mathcal{A} p, n \in \mathbb{N}$, and $\varepsilon>0$, there exists $P \in \mathcal{P}$ er such that $d_{u}(T, P)<\varepsilon+1 / n$.

The Rokhlin lemma lies in the basis of many areas of ergodic theory. The key concept related to this result is a tower constructed by an automorphism $T$ : a 
collection of disjoint sets $\left(A, T A, \ldots, T^{n-1} A\right)$ is called a tower (or $T$-tower) of height $n$ with base $A$.

The formulated above results can be generalized in several directions. Firstly, one can prove that every non-singular automorphism is also approximated by periodic automorphisms in the uniform topology [Cha-Fr] (the Rokhlin lemma for non-singular automorphisms), that is the set of periodic automorphisms, $\mathcal{P e r}$, is dense in $\left(\operatorname{Aut}(X, \mathcal{B}, \mu), d_{u}\right)$. Secondly, S. Alpern Alp, Alp-Pr 3 proved a multiple Rokhlin tower theorem for measure preserving and non-singular aperiodic automorphisms which shows that the space $X$ can be partitioned into a finite or infinitely countable number of towers of prescribed measures.

Theorem 2.3 (multiple Rokhlin tower lemma). For any $k \geq 2$, let $n_{1}, \ldots, n_{k}$ be relatively prime positive integers and let $p_{1}, \ldots, p_{k}$ be positive numbers such that $n_{1} p_{1}+\ldots+n_{k} p_{k}=1$. Then for any aperiodic $T \in \operatorname{Aut}_{0}(X, \mathcal{B}, \mu)$ there exists sets $B_{i} \in \mathcal{B}, i=1, \ldots, k$, with $\mu\left(B_{i}\right)=p_{i}$ and such that $\left\{T^{j} B_{i}: i=1, \ldots, k ; j=\right.$ $\left.0, \ldots, n_{i}-1\right\}$ is a partition of $X$.

In the next sections, we will discuss the Rokhlin lemma type results in Borel and Cantor dynamics. It will be shown below that, like in ergodic theory, the underlying space is partitioned into a finite or countable collection of towers for these dynamics.

Let $G$ be a topological group. Following [Gl-Ki], we say that $G$ has the Rokhlin property if there exists some $g \in G$ such that the conjugates of $g$ constitute a dense subset in $G$.

Theorem 2.4 (conjugacy lemma). Let $T \in A u t(X, \mathcal{B}, \mu)$ be an aperiodic automorphism. Then:

(1) $\left\{S T S^{-1}: S \in A u t(X, \mathcal{B}, \mu)\right\}$ is dense in $\mathcal{A p}$ with respect to the uniform topology;

(2) $\left\{S T S^{-1}: S \in \operatorname{Aut}(X, \mathcal{B}, \mu)\right\}$ is dense in $\operatorname{Aut}(X, \mathcal{B}, \mu)$ with respect to the weak topology.

These results are due to Halmos $\mathrm{Hal} 2$ for measure preserving automorphisms. Theorem 2.4 was generalized to the group $\operatorname{Aut}(X, \mathcal{B}, \mu)$ in $\mathrm{Ch}-\mathrm{Kak}$ and $\mathrm{Fr}$. Also the case of an infinite $\sigma$-finite invariant measure was considered in Ch-Kak. Note that these statements are very useful for proving the density and $G_{\delta}$-ness of various classes of automorphisms.

2.3. Classes of automorphisms. In this part, we will consider the following classes of automorphisms of a measure space: periodic, aperiodic, ergodic, mixing and weakly mixing, zero entropy, and of a finite rank.

As mentioned above, periodic automorphisms form a dense subset in $\operatorname{Aut}(X, \mathcal{B}, \mu)$ with respect to the uniform and weak topologies:

$$
\overline{\mathcal{P} e r}^{d_{u}}=\overline{\mathcal{P} e r}^{d_{w}}=\operatorname{Aut}(X, \mathcal{B}, \mu) .
$$

Furthermore, the following two simple facts hold for the set of aperiodic automorphisms: (1) the set $\mathcal{A} p$ is a closed nowhere dense subset in $\operatorname{Aut}(X, \mathcal{B}, \mu)$ with respect to the uniform topology; (2) the set $\mathcal{A} p$ is dense in $\operatorname{Aut}(X, \mathcal{B}, \mu)$ with respect to $d_{w}$. Clearly, the first result is an immediate consequence of the Rokhlin lemma. On the other hand, the second statement follows from the Rokhlin property. 
Recall that a set of a topological space is called generic (or typical) if it contains a dense $G_{\delta}$ subset.

In Hal 1, Halmos answered the very important question about genericity of ergodic transformations.

THEOREM 2.5. The set of ergodic automorphisms is a dense $G_{\delta}$ subset of $A u t_{0}(X, \mathcal{B}, \mu)$ with respect to the weak topology and a nowhere dense subset in the uniform topology.

In the paper Ch-Kak, this result was generalized to the group $\operatorname{Aut}(X, \mathcal{B}, \mu)$ and to the group $\operatorname{Aut}_{0}(X, \mathcal{B}, \nu)$ where $\nu$ is an infinite $\sigma$-finite measure equivalent to $\mu$. Furthermore, the Choksi-Kakutani result on genericity of ergodics was improved in Ch-Haw-Pr by showing that the ergodic type $I I I_{1}$ automorphisms form a dense $G_{\delta}$ subset in $\left(\operatorname{Aut}(X, \mathcal{B}, \mu), d_{w}\right)$.

In Ro 1, it was proved that finite measure preserving ergodic automorphisms constitute a dense $G_{\delta}$ subset in $\mathcal{A} p$ with respect to the uniform topology. It can be easily seen that ergodics are dense in non-singular aperiodic automorphisms in $d_{u}$.

Let $K$ be a compact metric space. A Borel probability measure $\mu$ on $K$ is called an Oxtoby-Ulam measure (O-U measure) if it is non-atomic and positive on every non-empty open set. If $K$ is a connected compact manifold, then additionally the $\mathrm{O}-\mathrm{U}$ measure $\mu$ must be zero on the boundary $\partial K$. The following result, proved in O-U], states that a typical homeomorphism of $K$ is ergodic:

TheOrem 2.6. Let $\mu$ be an $O$-U measure on a compact manifold $K, \operatorname{dim} K \geq 2$. Then the set of $\mu$-ergodic homeomorphisms of $K$ is a dense $G_{\delta}$ subset in the group $H_{o m e o_{\mu}}(K)$ of all $\mu$-preserving homeomorphisms of $K$ with respect to the topology of uniform convergence. In particular, every compact manifold supports an ergodic homeomorphism.

It is worth to mention one of the main results from Alp-Pr 2. Namely, it was proved that the natural embedding of $\mathrm{Homeo}_{\mu}(K)$ into the space $\left(A u t_{0}(K, \mu), d_{w}\right)$ preserves $G_{\delta}$-ness.

THEOREM 2.7. Let $\mu$ be an $O-U$ measure on a compact connected manifold $K$. Let $\mathcal{V}$ be a conjugate invariant, weak topology $G_{\delta}$ subset subset of $\operatorname{Aut}(K, \mu)$, which contains an aperiodic automorphism. Then $\mathcal{V} \cap \mathrm{Homeo}_{\mu}(K)$ is a dense $G_{\delta}$ subset of $\mathrm{Homeo}_{\mu}(K)$ with respect to the topology of uniform convergence.

The statistical (asymptotical) properties of an ergodic automorphism are, in general, rather poor. The dynamical systems generated by mixing and weakly mixing automorphisms have more developed statistical properties. The study of such automorphisms was begun in the papers $\mathrm{Hal}$ 2, Hal 3, Ro 1, Ro 2. Now there exist a large number of articles devoted to mixing and weakly mixing automorphisms. We mention here only the classical results.

THEOREM 2.8. (1) The set of weakly mixing automorphisms forms a dense $G_{\delta}$ subset in $A_{u t}(X, \mathcal{B}, \mu)$ with respect to the weak topology;

(2) the set of mixing automorphisms is a meager subset of $A u t_{0}(X, \mathcal{B}, \mu)$ with respect to the uniform topology.

Another crucial notion of ergodic theory is entropy discovered by Kolmogorov and studied later in works by Rokhlin, Sinai, Ornstein, and many others. In [Ro 3], the following generic set was found: 
TheOREM 2.9. Automorphisms of zero entropy form a dense $G_{\delta}$ subset of Aut $_{0}(X, \mathcal{B}, \mu)$ with respect to the weak and uniform topologies.

In the papers Kat-St 1 and Kat-St 2, a new powerful method, based on the Rokhlin lemma, was discovered. The authors called this method a cyclic approximation by periodic automorphisms. Katok and Stepin showed that the speed of approximation of an automorphism $T \in A u t_{0}(X, \mathcal{B}, \mu)$ by periodic ones determines ergodic, spectral, and mixing properties of $T$. In particular, the following result was proved:

THEOREM 2.10. The set of automorphisms admitting cyclic approximation with a fixed speed contains a dense $G_{\delta}$ subset in $A_{u t}(X, \mathcal{B}, \mu)$ with respect to $d_{w}$.

It follows from this theorem that the set of automorphisms of rank 1 is generic in $\left(A u t_{0}(X, \mathcal{B}, \mu), d_{w}\right)$.

In the space $\operatorname{Aut}(X, \mathcal{B}, \nu), \nu(X)=\infty$, one can also consider the class of (infinite) measure preserving automorphisms. In this settings, there exists a number of results on typical behavior of infinite measure preserving automorphisms. We mention the following facts proved in [Kre] and $\mathrm{Sa}$, respectively.

THEOREM 2.11. (1) Incompressible (conservative) automorphisms acting on an infinite measure space form a dense $G_{\delta}$ subset in the uniform and weak topologies. (2) Automorphisms with finite ergodic index form a meager set, but those with infinite index are generic in $\left(\operatorname{Aut}_{0}(X, \mathcal{B}, \nu), d_{w}\right), \nu(X)=\infty$.

Recently, Choksi and Nadkarni [Ch-N] proved that the set of non-singular automorphisms with infinite ergodic index is a dense $G_{\delta}$ subset in $\operatorname{Aut}(X, \mathcal{B}, \mu)$ with respect to the weak topology.

2.4. Subgroups of $A u t(X, \mathcal{B}, \mu)$ defined by an automorphism. To every automorphism $T$, we can associate the full group $[T]$ generated by $T$, the normalizer $N[T]$, and the centralizer $C(T)$. Here we consider topological properties of these groups. Notice that some of the results formulated below hold for more complicated groups of automorphisms than $\mathbb{Z}$.

THEOREM 2.12. (1) The full group $[T]$ of any automorphism $T \in \operatorname{Aut}(X, \mathcal{B}, \mu)$ is a closed nowhere dense subset of $\operatorname{Aut}(X, \mathcal{B}, \mu)$ in the uniform topology;

(2) the full group $[T]$ is a Polish group in the uniform topology;

(3) the full group $[T]$ of any ergodic finite measure preserving automorphism $T$ is a meager dense subset in $\operatorname{Aut}(X, \mathcal{B}, \mu)$ with respect to the weak topology;

(4) the full group $[T]$ of an ergodic automorphism $T$ is topologically simple in $d_{u}$;

(5) the full group $[T]$ is contractible in the uniform topology.

The first two results are simple exercises. In statement (3), the fact that $[T]$ is dense is also trivial, and the other part of the statement was observed by A. Kechris. The fact that for ergodic $T$ the full group $[T]$ has no uniformly closed normal subgroups follows from Dye (for type $I I$ automorphisms) and [B-Gol] (for type III automorphisms). The last statement was proved in Dan.

We note also that the notion of full group is extremely useful in the study of problems related to the orbit equivalence theory of ergodic non-singular automorphisms. As shown in G-P-S 2], full groups (and topological full groups) play a similar role in Cantor dynamics. 
Recall that on the normalizer $N[T]$ can be endowed with a topology generated by the metric $\rho$ Ha-Os]:

$$
\rho\left(R_{1}, R_{2}\right)=d_{w}\left(R_{1}, R_{2}\right)+\sum_{n \in \mathbb{Z}} 2^{-|n|} \frac{d_{u}\left(R_{1} T^{n} R_{1}^{-1}, R_{2} T^{n} R_{2}^{-1}\right)}{1+d_{u}\left(R_{1} T^{n} R_{1}^{-1}, R_{2} T^{n} R_{2}^{-1}\right)} .
$$

The results formulated in the following theorem are taken from Ha-Os (the first two statements) and from Dan (the last two statements).

THEOREM 2.13. Let $T$ be an ergodic non-singular automorphism. Then:

(1) $N[T]$ is a Polish space with respect to $\rho$;

(2) let $S \in \operatorname{Aut}(X, \mathcal{B}, \nu), \nu(X)=\infty$, and $\nu$ is $S$-invariant; then $R \in \mathbb{N}[S]$ belongs to the closure of $[S]$ in $(N[S], \rho)$ if and only if $\nu \circ R=\nu$;

(3) the normalizer $N[T]$, where $T$ is of type $I I$, is contractible with respect to $\rho$;

(4) if $T$ is an ergodic automorphism of type $I I I_{\lambda}$, then the fundamental group $\pi_{1}(N[T])=\mathbb{Z}$.

We finish this section with the weak closure theorem proved in Ki for rank 1 transformations.

TheOREM 2.14. Let $T$ be a rank 1 automorphism from $\operatorname{Aut}_{0}(X, \mathcal{B}, \mu)$ and let $C(T)$ denote the centralizer of $T$. Then ${\overline{\left\{T^{n}: n \in \mathbb{Z}\right\}}}^{d_{w}} \supset C(T)$.

Notice also that the centralizer $C(T)$ is closed in the weak topology.

\section{Topologies in Borel dynamics}

In this section, we define and study topologies on $\operatorname{Aut}(X, \mathcal{B})$ which are analogous to the weak and uniform topologies on $\operatorname{Aut}(X, \mathcal{B}, \mu)$. There exist many papers devoted to Borel dynamics but, as far as we know, topologies on $\operatorname{Aut}(X, \mathcal{B})$ were first studied in our papers [B-K 1], B-D-K 1], and [B-M].

3.1. The uniform and weak topologies on $\operatorname{Aut}(X, \mathcal{B})$. We first define two topologies on $A u t(X, \mathcal{B})$ and then consider the topological properties of the space $\operatorname{Aut}(X, \mathcal{B})$.

Given $S, T \in \operatorname{Aut}(X, \mathcal{B}, \mu)$, denote by $E(S, T)=\{x \in X \mid T x \neq S x\} \cup\{x \in$ $\left.X \mid T^{-1} x \neq S^{-1} x\right\}$.

Definition 3.1. ${ }^{3}$ The topologies $\tau$ and $p$ on $\operatorname{Aut}(X, \mathcal{B})$ are defined by the bases of neighborhoods $\mathcal{U}$ and $\mathcal{W}$, respectively. They are: $\mathcal{U}=\left\{U\left(T ; \mu_{1}, \ldots, \mu_{n} ; \varepsilon\right)\right\}$, $\mathcal{W}=\left\{W\left(T ; F_{1}, \ldots, F_{k}\right)\right\}$, where

$$
\begin{gathered}
U\left(T ; \mu_{1}, \ldots, \mu_{n} ; \varepsilon\right)=\left\{S \in \operatorname{Aut}(X, \mathcal{B}) \mid \mu_{i}(E(S, T))<\varepsilon, i=1, \ldots, n\right\}, \\
W\left(T ; F_{1}, \ldots, F_{k}\right)=\left\{S \in \operatorname{Aut}(X, \mathcal{B}) \mid S F_{i}=T F_{i}, i=1, \ldots, k\right\},
\end{gathered}
$$

In all the above definitions $T \in \operatorname{Aut}(X, \mathcal{B}), \mu_{1}, \ldots, \mu_{n} \in \mathcal{M}_{1}(X), F_{1}, \ldots, F_{k} \in \mathcal{B}$, and $\varepsilon>0$.

\footnotetext{
${ }^{3}$ In fact, we defined in B-D-K 1 more topologies on $A u t(X, \mathcal{B})$ but our main results concern mostly $\tau$ and $p$, so that we do not consider the remaining topologies in this paper.
} 
We call $\tau$ and $p$ the uniform and weak topologies, respectively. Our motivation is as follows. For a standard Borel space, we do not have a fixed Borel measure on the underlying space. Therefore, if we want to extend definitions (2.1) and (2.2) to $\operatorname{Aut}(X, \mathcal{B})$, we have to take into account the set $\mathcal{M}_{1}(X)$ of all Borel probability measures on $(X, \mathcal{B})$. To generalize the definition of $d_{w}$, we observe that if the symmetric difference of two Borel sets is arbitrarily small with respect to any $\mu \in$ $\mathcal{M}_{1}(X)$, then these sets must coincide.

Notice that if the set $E_{0}(S, T)=\{x \in X: S x \neq T x\}$ were used in (3.1) instead of $E(S, T)$, then we would obtain the topology equivalent to $\tau$.

Observe that the uniform topology $\tau$ is equivalent to $\tau^{\prime}$ which can be defined on $\operatorname{Aut}(X, \mathcal{B})$ similarly to the metric $d_{u}^{\prime}(2.3)$.

It is natural to consider two more topologies $\tau_{0}$ and $p_{0}$ on $\operatorname{Aut}(X, \mathcal{B})$ (they are some modifications of $\tau$ and $p$ ) by considering only continuous measures and uncountable Borel sets in Definition 3.1

Definition 3.2. The topologies $\tau_{0}$ and $p_{0}$ on $\operatorname{Aut}(X, \mathcal{B}, \mu)$ are defined by the bases of neighborhoods $\mathcal{U}_{0}=\left\{U_{0}\left(T ; \nu_{1}, \ldots, \nu_{n} ; \varepsilon\right)\right\}$ and $\mathcal{W}_{0}=\left\{W_{0}\left(T ; A_{1}, \ldots, A_{n}\right)\right\}$, respectively, where $U_{0}\left(T ; \nu_{1}, \ldots, \nu_{n} ; \varepsilon\right)$ and $W_{0}\left(T ; A_{1}, \ldots, A_{n}\right)$ are defined as in (3.1) and (3.2) with continuous measures $\nu_{i} \in \mathcal{M}_{1}(X)$ and uncountable Borel sets $A_{i}$ for $i=1, \ldots, n$.

Clearly, $\tau$ and $p$ are not weaker than $\tau_{0}$ and $p_{0}$, respectively.

Given an automorphism $T$ of $(X, \mathcal{B})$, we can associate a linear unitary operator $L_{T}$ on the Banach space $B(X)$ of all bounded Borel functions by $\left(L_{T} f\right)(x)=$ $f\left(T^{-1} x\right)$. Let $\tilde{p}$ be the topology induced on $\operatorname{Aut}(X, \mathcal{B})$ by the strong operator topology on bounded linear operators of $B(X)$. It was shown in [B-D-K 1] that the topologies $p$ and $\tilde{p}$ are equivalent. This fact corresponds to the well known result in ergodic theory (see Theorem 2.2) and is another justification of the name "weak topology" which is used to refer to $p$.

The following theorem proved in B-D-K 1 reveals some topological properties of $A u t(X, \mathcal{B})$ with respect to the topologies we have defined.

TheOREM 3.3. (1) $\operatorname{Aut}(X, \mathcal{B})$ is a Hausdorff topological group with respect to the topologies $\tau, \tau_{0}, p$, and $p_{0}$;

$(2)$ the sets $W\left(T ; F_{1}, \ldots ; F_{n}\right)$ are closed in $\operatorname{Aut}(X, \mathcal{B})$ with respect to $\tau$ and clopen with respect to $p$, that is $(A u t(X, \mathcal{B}), p)$ is a 0-dimensional topological group;

(3) $\operatorname{Aut}(X, \mathcal{B})$ is a complete non-separable group with respect to $\tau$ and $p$ in the sense that every Cauchy sequence of Borel automorphisms converges to a Borel automorphism;

(4) the group $\operatorname{Aut}(X, \mathcal{B})$ is totally disconnected in the uniform topology $\tau$;

$(5)$ the group $A u t(X, \mathcal{B})$ is path-connected in the topology $\tau_{0}$;

(6) the topology $p$ is equivalent to $p_{0}$;

(7) the topology $\tau$ is not comparable with $p$.

Let us give a few comments on the results from Theorem 3.3 Firstly, observe that, in contrast to the ergodic theory, the uniform and weak topologies are not comparable on $\operatorname{Aut}(X, \mathcal{B})$. Secondly, to see that that $(\operatorname{Aut}(X, \mathcal{B}), \tau)$ is totally disconnected, it suffices to notice the following facts: (i) the set of all automorphisms which have a fixed period at a fixed point is clopen in $\tau$ (see Theorem 3.6 (3) below) and (ii) $C t b l(X)$ is the unique normal subgroup of $A u t(X, \mathcal{B})$ which is not connected in its turn. On the other hand, the fact that the topological space $\left(\operatorname{Aut}(X, \mathcal{B}), \tau_{0}\right)$ 
is path-connected (proved in $[\mathrm{B}-\mathrm{M}]$ ) well corresponds to the results from [B-Gol, Dan, Kea mentioned in Theorem 2.1.

It is obvious that the topologies on $\operatorname{Aut}(X, \mathcal{B})$ are not defined by convergent sequences, however, it is useful for many applications to know criteria of convergence.

REMark 3.4. (1) $\left(T_{n}\right)$ converges to $S$ in $\tau$ if and only if $\forall x \in X \exists n(x) \in \mathbb{N}$ such that $\forall n>n(x), T_{n} x=S x$.

(2) $\left(T_{n}\right)$ converges to $S$ in $p$ if and only if for any Borel set $F, T_{n} F=T F$ for all sufficiently large $n$. In particular, $F$ can be a point from $X$. Therefore, we see that $p$-convergence implies $\tau$-convergence.

(3) Observe that the criterion of convergence (1) does not hold for the topology $\tau_{0}$.

3.2. The quotient group $\widehat{A u t}(X, \mathcal{B})$ and quotient topologies. Identifying Borel automorphisms from $A u t(X, \mathcal{B})$ which are different on an at most countable set, we obtain elements from the quotient group $\widehat{A u t}(X, \mathcal{B})=\operatorname{Aut}(X, \mathcal{B}) / \operatorname{Ctbl}(X)$. Observe that $C \operatorname{tbl}(X)$ is a closed normal subgroup with respect to the topologies $\tau, p$, and $\tau_{0}$ (see [B-M] and [B-D-K 1]). Denote by $\widehat{\tau}, \widehat{\tau_{0}}$ and $\widehat{p}$ the quotient topologies induced on $\widehat{\operatorname{Aut}}(X, \mathcal{B})$ by $\tau, \tau_{0}$ and $p$, respectively. Notice that the bases of the topologies $\widehat{\tau}$ and $\widehat{p}$ form by the sets $\widehat{U}\left(T ; \mu_{1}, \ldots, \mu_{n} ; \varepsilon\right)=U\left(T ; \mu_{1}, \ldots, \mu_{n} ; \varepsilon\right) \operatorname{Ctbl}(X)$ and $\widehat{W}\left(T ; F_{1}, \ldots, F_{m}\right)=W\left(T ; F_{1}, \ldots, F_{m}\right) C t b l(X)$, respectively, with continuous measures $\mu_{i}$ and uncountable Borel sets $F_{j}$. The class of automorphisms equivalent to a Borel automorphism $T$ we again denote by the same symbol $T$ and write $T \in \widehat{A u t}(X, \mathcal{B})$. This identification corresponds to the situation in ergodic theory when two automorphisms are identified if they are different on a set of measure 0 (mod-0-convention).

The following theorem clarifies some topological and algebraic properties of $\widehat{\operatorname{Aut}}(X, \mathcal{B})$.

THEOREM 3.5. (1) $\widehat{\operatorname{Aut}}(X, \mathcal{B})$ is a Hausdorff topological group with respect to the quotient topologies $\widehat{\tau}$ and $\widehat{p}$; the topologies $\widehat{\tau_{0}}$ and $\widehat{\tau}$ are equivalent on $\widehat{\operatorname{Aut}}(X, \mathcal{B})$; (2) $\widehat{\operatorname{Aut}}(X, \mathcal{B})$ is an algebraically simple group;

(3) the group $(\widehat{\operatorname{Aut}}(X, \mathcal{B}), \widehat{\tau})$ is path-connected.

The second statement was proved in Sh] and the other results were obtained in $[\mathrm{B}-\mathrm{M}]$. Observe that the identification of automorphisms different on an at most countable set improves considerably the topological structure of the quotient group. Apparently, one can conjecture the contractibility of $\widehat{\operatorname{Aut}}(X, \mathcal{B})$ in $\widehat{\tau}$. This would be parallel to the known result in the ergodic theory. We believe that the space $(\widehat{\operatorname{Aut}}(X, \mathcal{B}), \widehat{\tau})$ has the topological and algebraic structures similar to those of $\left(\operatorname{Aut}(X, \mathcal{B}, \mu), d_{u}\right)$.

3.3. Approximation results in $\operatorname{Aut}(X, \mathcal{B})$. In this section, we consider several natural classes of automorphisms and find their closures in the topologies we have defined above.

We first consider the sets of periodic and aperiodic Borel automorphisms. Let $T \in \operatorname{Aut}(X, \mathcal{B}, \mu)$. Then the space $X$ can be partitioned into a disjoint union of Borel $T$-invariant sets $X_{1}, X_{2}, \ldots, X_{\infty}$ where $X_{n}$ is the set of points with period 
$n$, and $X_{\infty}$ is the set where $T$ is aperiodic. Denote by $\mathcal{P e r}_{n}(x)$ the set of all automorphisms which have period $n$ at $x$. By definition, $T$ is in $\mathcal{P e r}_{n}$, the set of all Borel automorphisms of period $n$, if $X_{n}=X$. In other words,

$$
\mathcal{P e r}_{n}=\bigcap_{x \in X} \operatorname{Per}_{n}(x) \text {. }
$$

We say that $T \in \mathcal{P} e r_{0}$ if there exists $N \in \mathbb{N}$ such that $P^{N} x=x, x \in X$.

The following two theorems describe some topological properties of the sets of periodic and aperiodic automorphisms in $\operatorname{Aut}(X, \mathcal{B})$ (cf. (2.4)).

Theorem 3.6 (periodic automorphisms). (1) The set $\mathcal{P} e r_{0}$ is dense in $(\operatorname{Aut}(X, \mathcal{B}), \tau)$.

(2) $\mathcal{P}$ er is a closed nowhere dense subset in Aut $(X, \mathcal{B})$ with respect to $p$.

(3) For any $n \in \mathbb{N}$, the set $\mathcal{P e r}_{n}(x)(x \in X)$ is clopen with respect to the topologies $\tau$ and $p$.

(4) $\overline{\mathcal{P e r}}^{\tau}=\overline{\mathcal{P e r}}^{\tau}$.

(5) $\operatorname{Per}_{n}(n \in \mathbb{N})$ is closed with respect to $\tau$ and $p$.

Notice that statement (2) was proved in $[\mathrm{B}-\mathrm{M}$ and all other results are taken from $\mathrm{B}-\mathrm{D}-\mathrm{K} 1$. It is curious enough that the set of periodic automorphisms is rare in the topology $p$.

THEOREM 3.7 (aperiodic automorphisms). The set $\mathcal{A} p$ is closed and nowhere dense in $\operatorname{Aut}(X, \mathcal{B})$ with respect to $\tau$ and $p$.

Proof. We prove here that $\mathcal{A} p$ has no interior points in the topology $p$ (all other statements were obtained in B-D-K 1] $)$. Let $T \in \mathcal{A} p$ and let $W=W\left(T ; F_{1}, \ldots, F_{n}\right)$ be a $p$-neighborhood of $T$, where $\left(F_{1}, \ldots, F_{n}\right)$ is a partition of $X$. We need to show that $W$ contains a non-aperiodic automorphism. To do this, it suffices to change $T$ at some points and construct an automorphism $P \in W$ which has a periodic orbit. Fix a point $x \in X$ and consider the $T$-orbit of $x$. Then we can find two numbers $i, j \in \mathbb{Z}$ and two sets (say $F_{n_{1}}$ and $F_{n_{2}}$ ) such that $j \geq i+2$ and $T^{i} x, T^{j} x \in F_{n_{1}}$ and $T^{i+1} x, T^{j+1} x \in F_{n_{2}}$ (possibly, $n_{1}=n_{2}$ ). Define the automorphism $P$ as follows: $P T^{i} x=T^{j+1} x, P T^{j} x=T^{i+1} x$, and $P x=T x$ elsewhere. It is not hard to check that $P \in W$ and, clearly, $P \notin \mathcal{A} p$.

The next lemma is one of the main tools in the study of Borel automorphisms. The proof can be found in $\mathrm{Be}-\mathrm{Ke}$ or $[\mathrm{N}$. We formulate the lemma as in $\mathrm{B}-\mathrm{D}-\mathrm{K}$ 1].

Lemma 3.8. Let $T \in A u t(X, \mathcal{B}, \mu)$ be an aperiodic Borel automorphism of a standard Borel space $(X, \mathcal{B})$. Then there exists a sequence $\left(A_{n}\right)$ of Borel sets such that

(i) $X=A_{0} \supset A_{1} \supset A_{2} \supset \cdots$,

(ii) $\bigcap_{n} A_{n}=\emptyset$,

(iii) $A_{n}$ and $X \backslash A_{n}$ are complete $T$-sections, $n \in \mathbb{N}$,

(iv) for $n \in \mathbb{N}$, every point in $A_{n}$ is recurrent,

(v) for $n \in \mathbb{N}, A_{n} \cap T^{i}\left(A_{n}\right)=\emptyset, i=1, \ldots, n-1$,

(vi) for $n \in \mathbb{N}$, the base of every non-empty $T$-tower built by the function of the first return to $A_{n}$ is an uncountable Borel set.

A sequence of Borel sets $\left(A_{n}\right)$ satisfying Lemma 3.8 is called a vanishing sequence of markers. 
Recall that a Borel partition $\Xi=\left\{\xi_{i}: i \in I\right\}\left(|I| \leq \aleph_{0}\right)$ of $X$ is called a $K-R$ (Kakutani-Rokhlin) partition for an automorphism $T \in A u t(X, \mathcal{B})$ if all the $\xi_{i}$ 's are $T$-towers, $\xi_{i}=\left\{B_{i}, T B_{i}, \ldots, T^{m-1} B_{i}\right\}$, for some $B_{i} \in \mathcal{B}$. Set $B(\Xi)=\bigcup_{i \in I} B_{i}$.

The crucial importance of Lemma 3.8 may be partially explained by the following fact. It turns out that, having a vanishing sequence of markers, one can construct a sequence of Kakutani-Rokhlin partitions satisfying the following properties.

Proposition 3.9 (K-R partitions). Let $T$ be an aperiodic Borel automorphism of $(X, \mathcal{B})$. There exists a sequence of $K-R$ partitions $\left\{\Xi_{n}\right\}$ of $X$ whose elements generate the $\sigma$-algebra $\mathcal{B}$ and such that: (i) $\bigcap_{n} B\left(\Xi_{n}\right)=\emptyset$; (ii) $\Xi_{n}$ refines $\Xi_{n+1}$; and (iii) if $h\left(\xi_{i}\right)$ is the height of $\xi_{i}$, then $h_{n}=\min \left\{h(\xi): \xi \in \Xi_{n}\right\} \rightarrow \infty$ as $n \rightarrow \infty$.

Based on this result, we introduced in [B-D-K 1] the concept of Bratteli diagrams in the settings of Borel dynamics. It was shown that one can associate an ordered Bratteli diagram to every Borel aperiodic automorphism $T$ such that $T$ becomes isomorphic to the Vershik map acting on the space of infinite paths of the Bratteli diagram. Notice that the set of vertices at each level of the diagram (i.e., the number of towers in the K-R partitions) can be chosen finite, and the ordered diagram corresponding to $T$ has no maximal and minimal paths. It is worthy to recall that the notion of Bratteli diagrams was originally introduced in the theory of approximately finite $C^{*}$-algebras and then extensively studied by many authors in the setting of Cantor minimal systems (see, e.g. [H-P-S, G-P-S 1, Ma]).

We conclude this section with the following theorem [B-D-K 1].

Theorem 3.10. Let $T \in \operatorname{Aut}(X, \mathcal{B})$. Then: (1) the full group $[T](T \in$ Aut $(X, \mathcal{B}))$ is closed and nowhere dense in $\operatorname{Aut}(X, \mathcal{B})$ with respect to the topologies $\tau$ and $p$;

(2) $\mathcal{P e r}_{0} \cap[T]$ is $\tau$-dense in $[T]$ for each aperiodic $T$.

3.4. The Rokhlin lemma and Rokhlin property. The first result on approximation of any aperiodic Borel automorphism by periodic ones in the context of Borel dynamics was proved by Weiss in $\mathrm{W}$. Following $\mathrm{W}$, we call a Borel set $C$ completely positive with respect to an aperiodic automorphism $T$ if $\mu(C)>0$ for any $T$-nonsingular measure $\mu$. A Borel subset $A$ is called wandering for $T$ if $T^{i} A \cap A=\emptyset, i \neq 0$.

THEOREM 3.11. If $T$ is aperiodic Borel automorphism and $C$ is a Borel set such that $X \backslash C$ is completely positive, then for any prime $p$ there exists a Borel set $B$ with (i) $B, T B, \ldots, T^{p-1} B$ pairwise disjoint, and (ii) the set $C \backslash\left(\bigcup_{j=0}^{p-1} T^{j} B\right)$ is wandering.

To see how this theorem is related to the classical Rokhlin lemma, we recall the following fact. Let $\mathcal{W}(T)$ denote the $\sigma$-ideal of wandering sets with respect to $T$, and let $\mathcal{Q}(T)$ be the set of $T$-nonsingular measures on $\mathcal{B}$. Then

$$
\mathcal{W}(T)=\bigcap_{\mu \in \mathcal{Q}(T)} \mathcal{N}(T)
$$

where $\mathcal{N}(T)$ is the $\sigma$-ideal of $\mu$-null sets $[\mathrm{W}$.

Another approach to the problem of periodic approximation was applied by Nadkarni in $[\mathbb{N}$. In our notation, Nadkarni showed that for given $T \in \mathcal{A} p$, there exists a sequence of periodic automorphisms $\left(P_{n}\right)$ of $(X, \mathcal{B})$ such that $P_{n} \stackrel{\tau}{\longrightarrow} T, n \rightarrow$ 
$\infty$. Moreover, it the $P_{n}$ 's can be taken from $[T]$. It is clear now that this result is a direct consequence of Proposition 3.9

It turns out that the Rokhlin lemma can be also proved for any measure which is not related to a given aperiodic automorphism $T$ (see [B-D-K 1] for details).

Theorem 3.12 (Rokhlin lemma). Let $m \in \mathbb{N}$ and let $T$ be an aperiodic Borel automorphism of $(X, \mathcal{B})$. Then for any $\varepsilon>0$ and any measures $\mu_{1}, \ldots, \mu_{p}$ from $\mathcal{M}_{1}(X)$ there exists a Borel subset $F$ in $X$ such that $F, T F, \ldots, T^{m-1} F$ are pairwise disjoint and

$$
\mu_{i}\left(F \cup T F \cup \cdots \cup T^{m-1} F\right)>1-\varepsilon, \quad i=1, \ldots, p .
$$

To emphasize the obvious similarity between measurable dynamics and Borel dynamics, we give also two results on the Rokhlin property which were proved in B-M.

THEOREM 3.13 (Rokhlin property). (1) The action of $\widehat{A u t}(X, \mathcal{B})$ on itself by conjugation is topologically transitive with respect to the topology $\widehat{p}$. Moreover, $\left\{T^{-1} S T: T \in \widehat{A u t}(X, \mathcal{B})\right\}$ is dense in $(\widehat{\operatorname{Aut}}(X, \mathcal{B}), \widehat{p})$ for any $S \in \mathcal{S} m \cap \mathcal{A} p$.

(2) Let $T$ be an aperiodic automorphism of $(X, \mathcal{B})$, then $\left\{S^{-1} T S: S \in A u t(X, \mathcal{B})\right\}$ is dense in $(\mathcal{A p}, \tau)$.

We should also refer to the recent article Ke-Ros where the Rokhlin property is considered in the context of automorphism groups of countable structures.

3.5. Smooth automorphisms. We consider here the topological properties of the class $\mathcal{S} m$ of smooth automorphisms of a standard Borel space. This class is a natural extension of the class of periodic automorphisms.

We first note that the set $\mathcal{S} m$ is dense in $(A u t(X, \mathcal{B}), \tau)$. It follows from the fact that $\mathcal{P e r}$ is dense in $(\operatorname{Aut}(X, \mathcal{B}), \tau)$ and $\mathcal{P e r} \subset \mathcal{S} m$. On the other hand, by Theorem 3.7 the set $\mathcal{S} m \cap \mathcal{A} p$ is not dense in $\operatorname{Aut}(X, \mathcal{B})$ with respect to $\tau$ and $p$.

As shown in Theorem [3.6 the set of periodic automorphisms is not dense in $\operatorname{Aut}(X, \mathcal{B})$ with respect to $p$. Kechris conjectured that to obtain a dense subset in $(\operatorname{Aut}(X, \mathcal{B}), p)$, one needs to take the set of smooth automorphisms. The following theorem was proved in $\mathrm{B}-\mathrm{M}]$.

TheOrem 3.14. (1) $\overline{\mathcal{S} m}^{p}=A u t(X, \mathcal{B})$.

(2) $\mathcal{S} m \cap \mathcal{A} p$ is dense in $(\widehat{\operatorname{Aut}}(X, \mathcal{B}), \widehat{p})$.

(3) $\mathcal{S} m \cap \mathcal{A} p$ is dense in $\mathcal{A} p$ with respect to $p$.

3.6. The topology of uniform convergence. Let $(X, d)$ be a compact metric space. In this case, we can consider the group $A u t(X, \mathcal{B})$ of Borel automorphisms

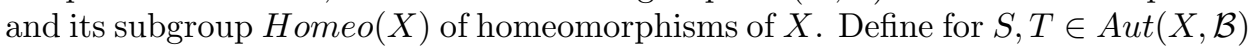
the topology of uniform convergence generated by the metric

$$
D(S, T)=\sup _{x \in X} d(S x, T x)+\sup _{x \in X} d\left(S^{-1} x, T^{-1} x\right) .
$$

Then $(\operatorname{Aut}(X, \mathcal{B}), D)$ is a complete metric space and $\operatorname{Homeo}(X)$ is $D$-closed in $\operatorname{Aut}(X, \mathcal{B})$. The case when $X(=\Omega)$ is a Cantor set is considered in Section 4 (see B-D-K 2, B-D-M for details). It is not hard to see that in Cantor dynamics the topology on $\operatorname{Homeo}_{(\Omega)}$ generated by $D$ is equivalent to the topology $p$ defined by clopen sets only. But in Borel dynamics the topologies $D$ and $p$ are different. Hence, it is interesting to find out which topological properties are preserved under 
embedding of $\operatorname{Homeo}(\Omega)$ into $\operatorname{Aut}(\Omega, \mathcal{B})$ endowed with the topologies $D$ and $p$. We note also that the topology generated by $D$ on $A u t(X, \mathcal{B})$ depends, in general, on the topological space $(X, d)$. Nevertheless, we think it is worthy to study the topological properties of $A u t(X, \mathcal{B})$ and $H o m e o(X)$ for a fixed compact (or Cantor) metric space $X$ because one can compare in this case these properties for the both groups.

The first problem we discuss for the metric $D$ is to find the closure of aperiodic Borel automorphisms of $X$ with respect to $D$.

Let $T$ be a Borel automorphism of $X$. Then $X$ is decomposed into the canonical $T$-invariant partition $\left(Y_{1}, Y_{2}, \ldots, Y_{\infty}\right)$ where $T$ has period $n$ on $Y_{n}$ and $T$ is aperiodic on $Y_{\infty}$ (see above 3.3). We call $T$ regular if all the sets $Y_{i}, 1 \leq i<\infty$, are uncountable.

Proposition 3.15. B-D-K 1] Suppose that $T \in A u t(X, \mathcal{B})$ is regular. Then for any $\varepsilon>0$ there exists $S \in \mathcal{A} p$ such that $D(S, T)<\varepsilon$.

Since $C t b l(X)$ is closed in $D$, we immediately obtain

COROLlary 3.16. B-D-K 1] The set of aperiodic automorphisms from $\widehat{A u t}(X, \mathcal{B})$ is dense with respect to the quotient topology $\widehat{D}$ of $D$.

To answer the question when a non-regular automorphism $T \in \operatorname{Aut}(X, \mathcal{B})$ belongs to $\overline{\mathcal{A p}}^{D}$, we need the following definition. We say that an automorphism $T$ is semicontinuous at $x \in X$ if for any $\varepsilon>0$ there exists $z \neq x$ such that $d(x, z)<\varepsilon$ and $d(T x, T z)<\varepsilon$.

TheOREM 3.17. B-D-K 1 Let $T$ be a non-regular Borel automorphism from Aut $(X, \mathcal{B})$ and let $\left(Y_{1}, Y_{2}, \ldots, Y_{\infty}\right)$ be the canonical partition associated to $T$. Denote by $Y_{0}$ the set $\bigcup_{i \in I} Y_{i}$ such that each $Y_{i}, i \in I$, is an at most countable set, $I \subset \mathbb{N}$. Then $T \in \overline{\mathcal{A p}}^{D}$ if and only if for every $x \in Y_{0}$ there exists $y \in \operatorname{Orb}_{T}(x)$ such that $T$ is semicontinuous at $y$.

To finish this section we present two more results on closures of some subsets of $A u t(X, \mathcal{B})$ proved in $\mathrm{B}-\mathrm{D}-\mathrm{K} 1$.

ThEOREM 3.18. The set $\mathcal{I}$ nc is a closed nowhere dense subset of $\mathcal{A} p$ with respect to the topology $p$.

Say that $T \in \mathcal{A} p \bmod (C t b l)$ if $T$ is aperiodic everywhere except an at most countable set.

Theorem 3.19. (1) $\overline{\mathcal{O d}}^{\tau}=\mathcal{A} p$ and $\overline{\mathcal{O}}^{\tau_{0}}=\mathcal{A} p \bmod (C t b l)$.

(2) $\overline{\mathcal{O d}}^{p} \subset \mathcal{I} n c$.

(3) $\overline{\mathcal{S m}}^{D} \supset \overline{\mathcal{O d}}^{D} \supset \mathcal{A} p$ assuming that $(X, d)$ is a compact metric space.

We note that full groups in the context of Borel dynamics were studied in many papers. We mention here J-Ke-L, Fel-Moo, Mer.

\section{Topologies in Cantor dynamics.}

In this section, we consider the uniform and weak topologies in the settings of Cantor dynamics. 
4.1. The weak and uniform topologies on Homeo $(\Omega)$. We define two topologies on Homeo $(\Omega)$ which are similar to the uniform and weak topologies used in Borel dynamics. Our primary goal is to study the global topological properties of $\mathrm{Homeo}_{(\Omega)}$ and compare these properties with those known for the groups $\operatorname{Aut}(X, \mathcal{B})$ and $\operatorname{Aut}(X, \mathcal{B}, \mu)$.

Definition 4.1. (i) The uniform topology $\tau$ on Homeo $(\Omega)$ is defined as the relative topology on $\operatorname{Homeo}(\Omega)$ induced from $(A u t(\Omega, \mathcal{B}), \tau)$. The base of neighborhoods is formed by

$$
\left.U\left(T ; \mu_{1}, \ldots, \mu_{n} ; \varepsilon\right)=\{S \in \operatorname{Homeo}(\Omega)) \mid \mu_{i}(E(S, T))<\varepsilon, i=1, \ldots, n\right\} .
$$

(ii) The topology $p$ is defined on $\operatorname{Homeo}(\Omega)$ by the base of neighborhoods

$$
W\left(T ; F_{1}, \ldots, F_{k}\right)=\left\{S \in \operatorname{Homeo}(\Omega) \mid S F_{i}=T F_{i}, i=1, \ldots, k\right\} .
$$

All measures in (4.1) are taken from $\mathcal{M}_{1}(X)$, and all the sets in (4.2) are assumed to be clopen.

Apparently, the most natural topology on the group Homeo( $\Omega)$ is the topology $D$ of uniform convergence which is generated by the metric $D(T, S)=$ $\sup _{x \in \Omega} d(T x, S x)+\sup _{x \in \Omega} d\left(T^{-1} x, S^{-1} x\right)$ ( $d$ is a metric on $\Omega$ compatible with the topology). Recall that this topology can be induced from $\operatorname{Aut}(\Omega, \mathcal{B})$ (3.4).

It is not hard to show that on the group $\operatorname{Homeo}(\Omega)$ the topologies $p$ and $D$ are equivalent and, as in the Borel case, the topologies $\tau$ and $p$ are not comparable (see details in B-D-K 2]).

Now we formulate several statements concerning topological and algebraic properties of Homeo $(\Omega)$.

TheOREM 4.2. (1) The group Homeo $(\Omega)$ is simple.

(2) Homeo $(\Omega)$ is a Hausdorff topological group with respect to the topologies $\tau$ and $p$.

(3) $(H o m e o(\Omega), p)$ is a 0-dimensional perfect Polish space and non-Archimedean topological group.

(4) The topological space $(H o m e o(\Omega), \tau)$ is totally disconnected.

(5) The group Homeo $(\Omega)$ is dense and non-closed in $($ Aut $(\Omega, \mathcal{B}), \tau)$.

Let us make a few comments on these results. The first statement is rather old and was proved in $\mathrm{An}$. The third result follows from the fact that the neighborhoods in (4.2) are closed, and the neighborhoods of the identity are subgroups of Homeo $(\Omega)$. To see that Homeo $(\Omega)$ is totally disconnected in $\tau$, we observe the following facts: (i) by (1), Homeo $(\Omega)$ has no normal subgroups, and (ii) there are nontrivial clopen subsets in $H_{o m e o}(\Omega)$ (for example, the set $\{S \in H o m e o(\Omega): S x=x\}$ is clopen with respect to $\tau)$. The last statement can be reformulated as follows: every Borel automorphism of $\Omega$ is approximated in $\tau$ by a homeomorphism. This is a form of Luzin's theorem. A similar approach can be also found in Alp-Pr 1 and Go-Ni-Wa .

Recall that $T_{n} \stackrel{\tau}{\longrightarrow} S \Longleftrightarrow \forall x \in \Omega \exists n(x) \in \mathbb{N}$ such that $\forall n>n(x), T_{n} x=S x$. On the other hand, $T_{n} \stackrel{p}{\longrightarrow} S \Longleftrightarrow \forall$ clopen $F \exists n(F)$ such that $\forall n>n(F), T_{n} F=$ $S F$, and $p$-convergence is equivalent to the uniform convergence, i.e., in the metric $D$. 
4.2. The Rokhlin lemma and Rokhlin property. We will discuss in this section the problem of approximation of aperiodic homeomorphisms by periodic ones. Recall that $\mathcal{P} e r$ denote the set of (pointwise) periodic homeomorphisms and $\mathcal{P e r} r_{0}$ is the set of periodic homeomorphisms with finite period.

We begin with the following result proved in B-D-M which asserts the existence of K-R partitions for aperiodic homeomorphisms.

THEOREM 4.3. Let $T$ be an aperiodic homeomorphism of $\Omega$. Given a positive integer $m \geq 2$, there exists a partition $\Xi=\left(\xi_{1}, \ldots, \xi_{k}\right)$ of $\Omega$ (aK-R partition) into a finite number of $T$-towers $\xi_{i}$ such that the height $h\left(\xi_{i}\right)$ of each tower is at least $m$.

The idea of the proof is the following. Given $m \in \mathbb{N}$, find a clopen partition of $\Omega=\bigcup_{i=1}^{q} U_{i}$ such that $T^{i} U_{j} \cap U_{j}=\emptyset$ for $i=0, \ldots, m-1$. Set $A_{1}=U_{1}$ and $A_{i}=U_{i} \backslash\left(\bigcup_{j=-m}^{m} T^{j}\left(A_{1} \cup \ldots \cup A_{i-1}\right)\right)$ for $i=2, \ldots, q$. Notice that the set $A=A_{1} \cup \ldots \cup A_{q}$ is clopen and meets every $T$-orbit at least once. Therefore for every $x \in A$ there is $l=l(x)>0$ such that $T^{l} x \in A$. The function of the first return defines a K-R partition $\Xi$ of $\Omega$ with base $A$ such that the height of every $T$-tower from $\Xi$ is at least $m$.

Based on this result, we prove a topological version of the Rokhlin lemma (the details are in B-D-M] ) which contains the following three results concerning approximation of aperiodic homeomorphisms by periodic ones.

THEOREM 4.4. (Rokhlin lemma) Let $T$ be an aperiodic homeomorphism of $\Omega$. (1) For $n \in \mathbb{N}, \varepsilon>0$, and any measures $\mu_{1}, \ldots, \mu_{k}$ from $\mathcal{M}_{1}(X)$, there exists a clopen subset $F$ in $\Omega$ such that $F, T F, \ldots, T^{n-1} F$ are pairwise disjoint and

$$
\mu_{i}\left(F \cup T F \cup \cdots \cup T^{n-1} F\right)>1-\varepsilon, \quad i=1, \ldots, k .
$$

(2) There exists a sequence of periodic homeomorphisms $\left\{P_{n}\right\} \subset \operatorname{Per}_{0} \cap[[T]]$ such that $P_{n} \stackrel{\tau}{\longrightarrow} T([T]]$ is the topological full group).

(3) Given $n \in \mathbb{N}$ and $\varepsilon>0$, there exists a $K-R$ partition $\Xi=\left(\xi_{1}, \ldots, \xi_{q}\right)$ of $\Omega$ as in Theorem 4.3 such that for $i=1, \ldots, k$

$$
\mu_{i}\left(\bigcup_{s=1}^{q} \bigcup_{j=0}^{h\left(\xi_{s}\right)-n}\right)>1-\varepsilon .
$$

To see that (3) holds, choose $m$ sufficiently large and repeat the construction used in Theorem 4.3 to find some $i$ from $(1, \ldots, m-n-1)$ such that $\mu_{j}\left(T^{i} A \cup \ldots \cup\right.$ $\left.T^{i+n} A\right)<\varepsilon$ for all $j=1, \ldots, k$. To obtain the K-R partition $\Xi$, apply again the above construction to the set $T^{i+n} A$ taken as a base of $\Xi$. The other statements are consequences of this result.

Remark. Observe that Theorem 4.4 allows one to construct a Bratteli diagram associated to an aperiodic homeomorphism. Moreover, one can show that every aperiodic homeomorphism of a Cantor set can be represented as the Vershik map acting on the path space of an ordered Bratteli diagram (see [M] for more details).

It turns out that, as in measurable and Borel dynamics, the Rokhlin property also holds in the group $H$ omeo $(\Omega)$.

THEOREM 4.5. (1) Let $T$ be an aperiodic homeomorphism of $\Omega$. Then the set $\left\{S^{-1} T S: S \in \operatorname{Homeo}(\Omega)\right\}$ is dense in $\mathcal{A} p$ with respect to the uniform topology $\tau$. 
(2) There exists a dense $G_{\delta}$ subset $\mathcal{E}$ of $(H o m e o(\Omega), p)$ such that $\left\{S^{-1} T S: S \in\right.$ Homeo $(\Omega)\}$ is p-dense in Homeo $(\Omega)$ for every $T \in \mathcal{E}$.

The first assertion was obtained in $B-\mathrm{D}-\mathrm{M}$ and the second one was proved in Gl-W 2. Observe that it is a nontrivial problem to point out explicitly a homeomorphism from the set $\mathcal{E}$. Indeed, if, say, $T$ is minimal then the closure of the conjugacy class of $T$ does not leave the set $\overline{\mathcal{M i n}}^{p}$. But the latter is a nowhere dense subset of $\operatorname{Homeo}(\Omega)$ in the topology $p$ (see below). We do not know whether there is an aperiodic homeomorphism such that its conjugates are dense in $(\operatorname{Homeo}(\Omega), p)$.

The Rokhlin property was also studied for some connected compact spaces. In Gl-W 2, the Rokhlin property was also established for any finite dimensional cube and the Hilbert cube. The local Rokhlin property was proved for circle homeomorphisms in A-Hu-Ken.

4.3. Approximation results in the group $\operatorname{Homeo}(\Omega)$. In this section, we consider various natural subsets of $\mathrm{Homeo}(\Omega)$ and find their closures. As mentioned above, Homeo $(\Omega)$ is not closed in $\operatorname{Aut}(X, \mathcal{B})$ in the uniform topology $\tau$, therefore the $\tau$-closure of a subset $Y \subset$ Homeo $(\Omega)$ does not belong to Homeo $(\Omega)$, in general. For convenience, we will use the following convention: $\bar{Y}^{\tau}:=\bar{Y}^{\tau} \cap \operatorname{Homeo}(\Omega)$ without further explanation.

Simple homeomorphisms. In the paper Gl-W 2, a new interesting class of homeomorphisms was defined. By definition, $S \in \mathrm{Homeo}(\Omega)$ is simple if it satisfies the following conditions.

(i) There exist non-empty clopen subsets $F_{j}$ and integers $r_{j} \geq 1, j=1, \ldots, k$, such that the collection $\left\{S^{i} F_{j}: i=0,1, \ldots, r_{j}, j=1, \ldots, k\right\}$ is pairwise disjoint and $S$ has period $r_{j}$ on $F_{j}$.

(ii) There exist clopen subsets $C_{s}, s=1, \ldots, l$, and, for each $s$, two disjoint periodic orbits $\left(y_{s}^{+}, S y_{s}^{+}, \ldots, S_{s}^{q_{s}^{+}-1} y_{s}^{+}\right)$and $\left(y_{s}^{-}, S y_{s}^{-}, \ldots, S^{q_{s}^{-}-1} y_{s}^{-}\right)$such that the sets $\left(S^{n} C_{s}: n \in \mathbb{Z}, s=1, \ldots, l\right)$ are pairwise disjoint and spiral towards the periodic orbits of $y_{s}^{+}$and $y_{s}^{-}$, that is $\lim _{n \rightarrow \pm \infty} \operatorname{dist}\left(S^{n} C_{s}, S^{n} y_{s}^{ \pm}\right)=0$

(iii) The space $\Omega$ may be represented as

$$
\Omega=\bigcup_{j=1}^{k} \bigcup_{i=0}^{r_{j}-1} S^{i} F_{j} \cup \bigcup_{s=1}^{l} \bigcup_{n \in \mathbb{Z}} S^{n} C_{s} \cup \bigcup_{s=1}^{l}\left[\left(y_{s}^{+}, \ldots, S^{q_{s}^{+}-1} y_{s}^{+}\right) \cup\left(y_{s}^{-}, \ldots, S^{q_{s}^{-}-1} y_{s}^{-}\right)\right]
$$

It follows from the definition that every simple homeomorphism $S$ contains a periodic part and there may exists a clopen set $C$ such that $T C \varsubsetneqq C$ or $C \varsubsetneqq T C$.

In Gl-W 2], the authors proved the following important result which was used to establish the Rokhlin property in $\operatorname{Homeo}(\Omega)$.

THEOREM 4.6. The set of simple homeomorphisms is dense in $($ Homeo $(\Omega), p)$.

Periodic and aperiodic homeomorphisms. As an immediate consequence of the Rokhlin lemma, we can find some topological properties of the sets of periodic and aperiodic homeomorphisms. The following two theorems was proved in B-D-K 2.

Theorem 4.7 (aperiodic). (1) $\mathcal{A} p$ is closed and nowhere dense in $($ Homeo $(\Omega), \tau)$.

(2) $\mathcal{A} p$ is dense in Homeo $(\Omega)$ with respect to the topology $p(\sim D)$. 
To prove (1), observe that $\mathcal{A} p$ is closed and Homeo $(\Omega) \backslash \mathcal{A} p$ is dense by Theorem 4.4 (3). The second assertion follows from the fact that every simple homeomorphism can be approximated in the topology $p$ by aperiodic ones. Then, we use Theorem 4.6 to complete the proof.

Theorem 4.8 (periodic). (1) $\overline{\mathcal{P e r}}^{\tau}=\operatorname{Homeo}(\Omega)$.

(2) The set $\overline{\mathcal{P e r}}^{p}$ is a proper subset in Homeo $(\Omega)$ containing minimal homeomorphisms.

(3) $\overline{\mathcal{P} e r}^{p}=\overline{\mathcal{P e r}}^{p}$.

Since the first result is new we give the proof of it. Observe that the fact that $\overline{\mathcal{P e r}}^{\tau} \supset \mathcal{A} p$ follows from the Rokhlin lemma (Theorem 4.4). Now, we give an idea how to approximate any $T \in \operatorname{Homeo}(\Omega)$ by elements from $\mathcal{P e r}_{0}$. For $T \in$ Homeo $(\Omega)$, consider a neighborhood $U=U\left(T ; \mu_{1}, \ldots, \mu_{k} ; \varepsilon\right)$. Take the canonical partition of $\Omega=X_{\infty} \cup \bigcup_{n \geq 1} X_{n}$ where $X_{n}$ is the set of points with period $n$ and $X_{\infty}$ consists of all aperiodic points. Notice that every $X_{n}=\bigcup_{i=0}^{n-1} T^{i} X_{n}^{0}, 0<n<\infty$, is a $T$-tower. By Proposition 3.9 we can find a K-R partition $\Xi=\left\{\xi_{1}, \xi_{2}, \ldots\right\}$ of $X_{\infty}$ such that $\mu_{l}\left(T^{-1} B(\Xi)\right)<\varepsilon / 4$ for $l=1, \ldots, k$ where $B(\Xi)$ is the base of $\Xi$. Find $n$ and $m$ such that $\mu_{l}\left(\xi_{1} \cup \ldots \xi_{n} \cup X_{1} \cup \ldots \cup X_{m}\right)>1-\varepsilon / 4$. Then, by regularity of the measures $\mu_{l}$, we can find in each $T$-tower $\xi_{i}$ and $X_{j}$, a closed subtower $\xi_{i}^{\prime}$ and $X_{j}^{\prime}$, resp., such that $\mu_{l}\left(\xi_{i} \backslash \xi_{i}^{\prime}\right)<\varepsilon(4 n)^{-1}$ and $\mu_{l}\left(X_{j} \backslash X_{j}^{\prime}\right)<\varepsilon(4 m)^{-1}, i=1, \ldots, n ; j=$ $1, \ldots, m$. Thus, we have obtained a finite number of disjoint closed $T$-towers such that $\mu_{l}\left(\xi_{1}^{\prime} \cup \ldots \cup \xi_{n}^{\prime} \cup X_{1}^{\prime} \cup \ldots X_{m}^{\prime}\right)>1-\varepsilon / 2$ and $\mu_{l}\left(T^{-1} B\left(\xi_{1}^{\prime} \cup \ldots \cup \xi_{n}^{\prime}\right)\right)<\varepsilon / 2$. For every $\xi_{i}^{\prime}$ and $X_{j}^{\prime}$, we find a clopen $T$-tower $\xi_{j}^{c o}$ and $X_{j}^{c o}$, resp., such that $\xi_{i}^{\prime}$ and $X_{j}^{\prime}$ are subtowers of $\xi_{j}^{c o}$ and $X_{j}^{c o}$ and $\mu_{l}\left(\bigcup_{i=1}^{n} T^{h\left(\xi_{i}^{c o}\right)} B\left(\xi_{i}^{c o}\right)\right)<\varepsilon / 2$. Moreover, we can choose all these clopen $T$-towers pairwise disjoint. Let $Q$ be the union of these clopen $T$-towers. Define $P \in \operatorname{Homeo}(\Omega)$ as follows: if $x \notin Q$, set $P x=x$; if $x \in Q$ and does not lie in the top level of a tower, set $P x=T x$, and for $x$ lying in the top level of a $T$-tower from $Q$ (say $\xi$ ), set $P x=T^{-h(\xi)+1} x$, where $h(\xi)$ is the height of $\xi$. Observe that $P \in U \cap \mathcal{P} e r_{0}$.

Statement (2) follows from the following observation: every dissipative homeomorphism does not belong to $\overline{\mathcal{P e r}}^{p}$. Recall that a homeomorphism $T$ is dissipative if there is a clopen set $F$ such that either $T F \varsubsetneqq F$ or $F \varsubsetneqq T F$. The fact that $\mathcal{M i n} \subset \overline{\mathcal{P e r}}_{0}^{p}$ was proved in B-D-K 2 where the closure of $\mathcal{P} e r_{0}$ in $p$ was found.

Odometers, minimal, and mixing homeomorphisms. This section contains our results on the description of closures of some natural subsets in $\mathrm{Homeo}(\Omega)$ with respect to the topologies $\tau$ and $p$. The notation $\mathcal{O} d, \mathcal{M i n}, \mathcal{M i x}$ was introduced in Section 1. Denote also by $\mathcal{T} t$ the set of topologically transitive homeomorphisms. Clearly, $\mathcal{O} d \subset \mathcal{M i n} \subset \mathcal{T} t \cap \mathcal{A} p \subset \mathcal{A} p$. As in ergodic theory, one can define the set $\mathcal{R}(1)$ of homeomorphisms of rank 1 but it is not hard to show that this set coincides with $\mathcal{O} d$. The following definition was introduced in B-D-K 2: A homeomorphism $T$ is called moving if for every proper clopen set $F$ each of the sets $F \backslash T F$ and $T F \backslash F$ is not empty. We denote the class of moving homeomorphisms by $\mathcal{M}$ ov. It is clear that $\operatorname{Per} \cap \mathcal{M}$ ov $=\emptyset$ and $\mathcal{M} o v \supset \mathcal{T} t \supset$ Min.

The next results were proved in $\mathrm{B}-\mathrm{D}-\mathrm{K} 2]$.

TheOrem 4.9. (1) The sets $\overline{\mathcal{M i n}}^{p}$ and $\overline{\mathcal{M i x}}^{p}$ are nowhere dense in $($ Homeo $(\Omega), p)$. 
(2) $\mathcal{O} d$ is a dense $G_{\delta}$-set in $\overline{\mathcal{M i n}}^{p}$.

(3) $\mathcal{O} d$ is an $F_{\sigma \delta}$-set in $\overline{\mathcal{M i n}}^{\tau}$.

(4) $\overline{\mathcal{O d}}^{\tau}=\overline{\mathcal{M i n}}^{\tau}=\overline{\mathcal{T} t}^{\tau} \cap \mathcal{A} p=\mathcal{A} p$ and $\overline{\mathcal{O d}}^{p}=\overline{\mathcal{M i n}}^{p}=\overline{\mathcal{T}}^{p}=\mathcal{M o v}$.

(5) $\mathcal{M i x} \cap \overline{\mathcal{M i n}}^{p}$ is nowhere dense in $\left(\overline{\mathcal{M i n}}^{p}, p\right)$.

In Gl-W 2, the following interesting theorem was proved.

TheOREM 4.10. (1) For a compact metric space $K$, the set of homeomorphisms having zero entropy is a $G_{\delta}$ subset in $H$ omeo $(K)$ in the topology of uniform convergence.

(2) The set of homeomorphisms having zero entropy is a dense $G_{\delta}$ subset in (Homeo $(\Omega), p)$.

(3) The set of homeomorphisms of the Hilbert cube having infinite topological entropy contains a dense $G_{\delta}$ subset in the topology of uniform convergence. This is also true for a finite dimensional cube.

Subgroups of Homeo $(\Omega)$ generated by a homeomorphism. In this section, we discuss topological properties of full groups, normalizers, and centralizers of Cantor systems.

Let $T$ be an aperiodic homeomorphism. It is easy to prove that the full group $[T]$ is closed and nowhere dense in $(\operatorname{Homeo}(\Omega), \tau)$. On the other hand, $[T]$ is not closed in $(\operatorname{Homeo}(\Omega), p)$, but $[T]$ is still nowhere dense.

The following theorem asserts that the full group of a minimal homeomorphism $T$ is separable since the topological full group is a dense countable subgroup in $[T]$ in the topology $\tau$. Notice that this result is not true, in general, with respect to the topology $p$. We find the class of minimal homeomorphisms for which the $p$-closures of $[[T]]$ and $[T]$ coincide. We say that a minimal homeomorphism $T$ is saturated if for any two clopen subsets $A$ and $B$ with $\mu(A)=\mu(B)$ for every $T$-invariant measure $\mu$ there is $\gamma \in[[T]]$ such that $\gamma(A)=B$ [B-K 1]. Observe that the class of saturated homeomorphisms contains odometers and does not contain the Chacon homeomorphisms. By Theorem 4.9 (2), the class of saturated homeomorphisms is generic in $\mathcal{M o v}$ with respect to $p$.

THEOREM 4.11. Let $T$ be a minimal homeomorphism of $\Omega$.

(1) The topological full group $[[T]]$ is $\tau$-dense in $[T]$.

(2) $\overline{[T]}^{p}=\left\{S \in \operatorname{Homeo}(\Omega): \mu \circ S=\mu\right.$ for all $\left.\mu \in M_{1}(T)\right\}$.

(3) $T$ is saturated if and only if $\overline{[[T]]}^{p}=\overline{[T]}^{p}$.

(4) If $T$ is of rank 1 (odometer), then $C(T)={\overline{\left\{T^{n}: n \in \mathbb{Z}\right\}}}^{p}$.

The first and third statements are proved in $\mathrm{B}-\mathrm{K} 1]$. The second result is taken from G-P-S 2, and (4) is a rather simple observation noticed in B-D-K 2.

For a homeomorphism $T \in \operatorname{Homeo}_{(\Omega)}$, let $\mathcal{P e r}_{0}(T)$ denote the set $\mathcal{P e r}_{0} \cap[[T]]$. Although $\mathcal{P e r}_{0}$ is not dense in $(\operatorname{Homeo}(\Omega), p)$, the following result holds. The first statement follows from Theorem $4.8(1)$, and (2) was proved in B-D-K 2].

TheOREM 4.12. (1) Let $T$ be a homeomorphism of $\Omega$, then $\overline{\mathcal{P e r}}(T)^{\tau}=[T]$. (2) Let $T$ be a minimal homeomorphism of $\Omega$, then $\overline{\mathcal{P e r}}(T)^{p} \supset[[T]]$.

Let $T$ be a homeomorphism of a Cantor set $\Omega$. Then $T$ can be also considered as a Borel automorphism of $(\Omega, \mathcal{B})$. Therefore, one can define two full groups $[T]_{C}$ and $[T]_{B}$ :

$$
[T]_{C}=\left\{S \in \operatorname{Homeo}(\Omega): S x \in\left\{T^{n} x \mid n \in \mathbb{Z}\right\} \forall x \in \Omega\right\},
$$




$$
[T]_{B}=\left\{S \in A u t(\Omega, \mathcal{B}): S x \in\left\{T^{n} x \mid n \in \mathbb{Z}\right\} \forall x \in \Omega\right\}
$$

where $C$ and $B$ stand for Cantor and Borel. Obviously, $[[T]]_{C} \subset[T]_{C} \subset[T]_{B}$ and $[T]_{B}$ is closed in $A u t(\Omega, \mathcal{B})$ with respect to $\tau$. Recall that by Theorem 4.11 (1) we have that for a minimal homeomorphism $T$,

$$
{\overline{[[T]]_{C}}}^{\tau} \cap \operatorname{Homeo}(\Omega)=[T]_{C} .
$$

The problem of finding of the closure of $[[T]]_{C}$ in $\operatorname{Aut}(\Omega, \mathcal{B})$ with respect to $\tau$ was solved in B-D-K 2] where the following result was obtained.

THEOREM 4.13. Let $T$ be a minimal homeomorphism of a Cantor set $\Omega$. Then ${\overline{[[T]]_{C}}}^{\tau}=[T]_{B}$.

This theorem has been recently generalized to any aperiodic homeomorphism $T$ by Medynets.

We finish this section by considering a topology on the normalizer $N[T]$ of

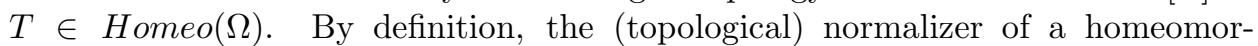
phism $T \in \operatorname{Homeo}(\Omega)$ is the group $N[T]=\left\{S \in \operatorname{Homeo}(\Omega): S\left(\operatorname{Orb}_{T}(x)\right)=\right.$ $\operatorname{Orb}_{T}(S x)$ for all $\left.x \in X\right\}$. In B-K 2, the following topology on $N[T]$ was introduced: for $R \in N[T]$, Borel probability measures $\mu_{1}, \ldots, \mu_{k}$, and $\varepsilon>0$ define $V\left(R ; \mu_{1}, \ldots, \mu_{k} ; \varepsilon\right)=\left\{P \in N[T]: D(R, P)<\varepsilon\right.$ and $\mu_{i}\left(E\left(R T^{n} R^{-1}, P T^{n} P^{-1}\right)\right)<$ $\varepsilon, i=1, \ldots, k, n \in \mathbb{Z}\}$. The sets $\left\{V\left(R ; \mu_{1}, \ldots, \mu_{k} ; \varepsilon\right)\right\}$ form a base of a topology $\lambda$. The following result is due to $\mathrm{B}-\mathrm{K} 2]$.

TheOrem 4.14. $(N[T], \lambda)$ is a complete separable topological group.

\section{Comparison of topological properties of transformation groups.}

In this section, we discuss the results from Sections 2 - 4. The reader can see that many of the formulated theorems are similar for measurable, Borel, and Cantor dynamics. This observation shows that the three dynamics have common properties in the topologies we have studied.

We first consider the groups $\operatorname{Aut}(X, \mathcal{B}, \mu), \operatorname{Aut}(X, \mathcal{B})$, and Homeo $(\Omega)$ with respect to the uniform topology and summarize their topological properties in the following table.

\section{Uniform topology}




\begin{tabular}{|c|c|c|c|c|c|}
\hline Group & $\operatorname{Aut}(X, \mathcal{B}, \mu)$ & \multicolumn{2}{|c|}{$\overline{A u t}(X, \mathcal{B})$} & $\widehat{A u t}(X, \mathcal{B})$ & Homeo $(\Omega)$ \\
\hline Topology & $d_{u}$ & $\tau$ & $\tau_{0}$ & $\widehat{\tau}$ & $\tau$ \\
\hline Connectedness & Yes & $\begin{array}{l}\text { totally } \\
\text { discon- } \\
\text { nected }\end{array}$ & Yes & Yes & $\begin{array}{l}\text { totally dic- } \\
\text { sonected }\end{array}$ \\
\hline $\begin{array}{l}\text { Path- } \\
\text { connectedness }\end{array}$ & Yes & $\mathrm{No}$ & Yes & Yes & No \\
\hline Contractibility & Unknown & $\mathrm{No}$ & Unknown & Unknown & No \\
\hline $\begin{array}{l}\text { Rokhlin } \\
\text { lemma and } \\
\text { Rokhlin prop- } \\
\text { erty }\end{array}$ & holds & holds & holds & holds & holds \\
\hline $\mathcal{P} e r$ & dense & dense & dense & dense & dense \\
\hline $\mathcal{A} p$ & $\begin{array}{l}\text { closed } \\
\text { nowhere } \\
\text { dense }\end{array}$ & $\begin{array}{l}\text { closed } \\
\text { nowhere } \\
\text { dense }\end{array}$ & $\begin{array}{l}\text { nonclosed } \\
\text { nowhere } \\
\text { dense }\end{array}$ & $\begin{array}{l}\text { closed } \\
\text { nowhere } \\
\text { dense }\end{array}$ & $\begin{array}{l}\text { closed } \\
\text { nowhere } \\
\text { dense }\end{array}$ \\
\hline Dense subsets & $\begin{array}{l}\overline{\mathcal{E} r g}^{d u}=\mathcal{A} p \\
\overline{\mathcal{O} d}^{d}=\mathcal{A} p\end{array}$ & $\overline{\mathcal{O} d}^{\tau}=\mathcal{A} p$ & $\overline{\overline{\mathcal{O d}}}^{\tau}=\mathcal{A} p$ & $\overline{\mathcal{O} d}^{\tau}=\mathcal{A} p$ & $\begin{array}{l}\overline{\mathcal{M i n}}^{\tau}=\mathcal{A} p \\
\overline{\mathcal{O d}}^{\tau}=\mathcal{A} p\end{array}$ \\
\hline
\end{tabular}

It is seen that if the topological groups from the table are connected then they are path-connected. The Rokhlin lemma and Rokhlin property hold for all these groups. Recall that by the Rokhlin property in the uniform topology we mean the density of conjugates of any aperiodic transformation in $\mathcal{A} p$. Another common property for these groups is the density of periodic transformations. This fact allows one to approximate every transformation by periodic ones. On the other hand, the set of aperiodic is closed and nowhere dense in all the dynamics. However, inside of the set of aperiodic transformations, we can find dense subsets consisting of transformations which are of "ergodic" type, i.e. odometers, minimal homeomorphisms. As seen from the table and obtained results, the best analogue of the space $\left(\operatorname{Aut}(X, \mathcal{B}, \mu), d_{u}\right)$ in Borel dynamics is apparently the quotient group $(\widehat{A u t}(X, \mathcal{B}), \widehat{\tau})\left(\right.$ or $\left.\left(A u t(X, \mathcal{B}), \tau_{0}\right)\right)$. Note that we have not considered properties of Homeo $(\Omega)$ with respect to $\tau_{0}$. We think it may be interesting to do this.

\section{Weak topology}

A similar comparison of these groups with respect to the weak topologies, $d_{w}$ and $p$, shows that they do not have much in common in contrast to the uniform topology. Nevertheless, we can point out some common features. Firstly, the set of aperiodic transformations is dense for each of these groups in the weak topology. Secondly, the Rokhlin property holds in $\operatorname{Aut}(X, \mathcal{B}, \mu), \widehat{\operatorname{Aut}}(X, \mathcal{B})$, and Homeo $(\Omega)$. Moreover, the following two facts hold: (i) the transformations of zero entropy are typical for $\operatorname{Aut}(X, \mathcal{B}, \mu)$ and $H o m e o(\Omega)$, (ii) rank 1 automorphisms are typical in $\operatorname{Aut}_{0}(X, \mathcal{B}, \mu)$ and odometers (or rank 1 homeomorphisms) are typical in the space Mov, the closure of all minimal homeomorphisms. Based on Theorem 4.9] one can suppose that the set Mov endowed with the topology $p$ is an analogue of the space $\left(\operatorname{Aut}(X, \mathcal{B}, \mu), d_{w}\right)$. On the other hand, the group $\operatorname{Aut}(X, \mathcal{B}, \mu)$ is contractible in the weak topology, but $(\widehat{A u t}(X, \mathcal{B}), \widehat{p})$ and $(\operatorname{Homeo}(\Omega), p)$ are totally disconnected topological spaces. 
For a Cantor set $\Omega$, the topology of uniform convergence defined by the metric $D$ and the topology $p$ are equivalent. If $(X, d)$ is a compact metric space then the topology $D$ and $p$ on $A u t(X, \mathcal{B})$ are different. It is interesting to compare topological properties of $(\operatorname{Homeo}(\Omega), p)$ and $(A u t(X, \mathcal{B}), D)$. We see that the set $\mathcal{A} p$ of periodic transformations is dense in $\left(\operatorname{Aut}(X, \mathcal{B}, \mu), d_{w}\right),(\operatorname{Homeo}(\Omega), p)$, and "almost" dense in $(\operatorname{Aut}(X, \mathcal{B}), D)$. We hope that the further study of possible relations between these topologies will lead to new interesting results.

\section{References}

[A-Hu-Ken] E. Akin, M. Hurley, J.A. Kennedy, Dynamics of Topologically Generic Homeomorphisms, Memoirs of the AMS, 164, 2003.

[Alp] S. Alpern, Return times and conjugates of an aperiodic transformation, Erg. Th. Dyn. Syst., 1 (1981), $135-143$.

[Alp-Pr 1] S. Alpern and V.S. Prasad, Typical Dynamics of Volume Preserving Homeomorphisms, Cambridge University Press, 2000.

[Alp-Pr 2] S. Alpern and V.S. Prasad, Properties generic for Lebesgue space automorphisms are generic for measure preserving manifold homeomorphisms, Erg. Th. Dyn. Syst., 22 (2002), $1587-1620$.

[Alp-Pr 3] S. Alpern and V.S. Prasad, Return times for nonsingular measurable transformations, J. Math. Anal. Appl., 152 (1990), 470 - 484

[An] R.D. Anderson, The algebraic simplicity of certain groups of transformations, Amer. J. Math., 80 (1958), 955 - 963.

[Be-Ke] H. Becker and A. Kechris, The Descriptive Set Theory of Polish Group Actions, London Math. Soc., Lecture Notes Series, 232, Cambridge University Press, 1996, xii + 136pp.

[B-Gol] S. Bezuglyi, V.Ya. Golodets, Topological properties of full groups of automorphisms of a space with a measure, (Russian) Sibirsk. Mat. Zh., 21 (1980), 3-13.

[B-D-K 1] S. Bezuglyi, A.H. Dooley, and J. Kwiatkowski, Topologies on the group of Borel automorphisms of a standard Borel space, arXiv:math.DS/0410506

[B-D-K 2] S. Bezuglyi, A.H. Dooley, and J. Kwiatkowski, Topologies on the group of homeomorphisms of a Cantor set, arXiv:math.DS/0410507

[B-D-M] S. Bezuglyi, A.H. Dooley, and K. Medynets, The Rokhlin lemma for homeomorphisms of a Cantor set, Proc AMS (to appear), arXiv:math.DS/0410505

[B-K 1] S. Bezuglyi, J. Kwiatkowski, The topological full group of a Cantor minimal system is dense in the full group, Topol. Methods in Nonlinear Anal., 16 (2000), 371 - 397.

[B-K 2] S. Bezuglyi, J. Kwiatkowski, Topologies on full groups and normalizers of Cantor minimal systems, Math. Physics , Analysis, and Geometry, 9 (2002), No.3, 1 - 10.

[B-M] S. Bezuglyi, K. Medynets, Smooth automorphisms and path-connectedness in Borel dynamics, Indag. Math. (to appear), arXiv:math.DS/0410504

[C-F-S] I. Cornfeld, S. Fomin, Ya. Sinai, Ergodic Theory, Grundlehren der mathematischen Wissenschaften 245, Springer-Verlag, 1982.

[Cha-Fr] R.V. Chacon, N.A. Friedman, Approximation and invariant measures, Z. Wahrscheinlichkeitstheorie und Verw. Gebiete, 3 (1965), 286-295.

[Ch-Haw-Pr] J.R. Choksi, J. Hawkins, . Prasad, Abelian cocycles for nonsingular ergodic transformations and the genericity of type $I I I_{1}$ transformations, Monatsh. Math., 103 (1987), 187-205.

[Ch-Kak] J.R. Choksi, S. Kakutani, Residuality of ergodic measurable transformations and of ergodic transformations which preserve an infinite measure, Ind. Univ. Math. J., 28 (1979), $453-469$.

[Ch-N] J.R. Choksi, M.G. Nadkarni, Genericity of nonsingular transformations with infinite ergodic index, Coll. Math., 84/85 (2000), 195 - 201.

[Ch-Pr] J.R. Choksi, V. Prasad, Approximation and Baire category theorems in ergodic theory, Measure Theory and its Applications, Lecture Notes in Mathematics, 1033 (1983), 94 - 113.

[Dan] A.I. Danilenko, The topological structure of Polish groups and groupoids of measure space transformations, Publ. RIMS, Kyoto Univ., 31 (1995), 913 - 940.

[Dye] H.A. Dye, On groups of measure preserving transformations. II, Amer. J. Math., 851963 $551-576$. 
[E 1] S.J. Eigen, On the simplicity of the full group of ergodic transformations, Isr. J. Math., 40 (1981), $345-349$.

[E 2] S.J. Eigen, The group of measure preserving transformations of $[0,1]$ has no outer automorphisms, Math. Ann., 259 (1982), 259 - 270.

[Fel-Moo] J. Feldman, C.C. Moore, Ergodic equivalence relations, cohomology, and von Neumann algebras. I, II, Trans. Amer. Math. Soc., 234 (1977), 289 - 359.

[Fr] N.A. Friedman, Introduction to Ergodic Theory, Van Nostrand Reinhold, New York, 1970.

[G-P-S 1] T. Giordano, I. Putnam, and C. Skau, Topological orbit equivalence and $C^{*}$-crossed products, J. reine angew. Math., 469 (1995), 51 - 111.

[G-P-S 2] T. Giordano, I. Putnam, and C. Skau, Full groups of Cantor minimal systems, Israel. J. Math., 111 (1999), 285 - 320.

[G-P-S 3] T. Giordano, I. Putnam, and C. Skau, Affable equivalence relations and orbit structure of Cantor dynamical systems, Erg. Th. Dyn. Syst., 24 (2004), $441-475$.

[Gl-Ki] E. Glasner,J. King, A zero-one law for dynamical properties, Topological dynamics and applications (Minneapolis, MN, 1995), 231-242, Contemp. Math., 215, Amer. Math. Soc., Providence, RI, 1998.

[Gl-W 1] E. Glasner, B. Weiss, Weak orbit equivalence of Cantor minimal systems, Intern. J. Math.,6 (1995), 569 - 579.

[Gl-W 2] E. Glasner and B. Weiss, The topological Rohlin property and topological entropy, Amer. J. Math., 123 (2001), 1055 - 1070.

[Go-Ni-Wa] G. Goffman, T. Nishiura, D. Waterman, Homeomorphisms in Analysis, Mathematical Surveys and Monographs, vol. 54, 1998.

[Ha-Os] T. Hamachi, M. Osikawa, Ergodic groups of automorphisms and Krieger's theorems, Seminar on Math. Sci., Keio Univ., 3 (1981), 1-113.

[Hal 1] P. Halmos, Approximation theories for measure preserving transformations, Trans. Amer. Math. Soc., 55 (1944), 1-18.

[Hal 2] P. Halmos, In general, a measure preserving transformation is not mixing, Ann. Math., 45(2) (1944), 786-792.

[Hal 3] P. Halmos, Lectures on Ergodic Theory, The Mathematical Society of Japan, Publications of the Mathematical Society of Japan, no. 3, 1956, vii+99 pp.

[H-P-S] R.H. Herman, I. Putnam, and C. Skau, Ordered Bratteli diagrams, dimension groups, and topological dynamics, Intern. J. Math., 3 (1992), 827 - 864.

[Hj] G. Hjorth, Classification and Orbit Equivalence Relation, AMS, 2000.

[IT] A. Ionescu Tulcea, On the category of certain classes of transformations in ergodic theory, Trans. Amer. Math. Soc., 114 (1965), $261-279$.

[J-Ke-L] S. Jackson, A. Kechris, A. Louveau, Countable equivalence relations, J. Math. Logic, 2(1) (2002), $1-80$.

[Kat-St 1] A. Katok, A. Stepin, Approximation in ergodic theory (Russian), Usp. Mat. Nauk, 22 (5) (1967), 81 - 106 (Engl. Transl. 'Russian Math. Surveys', 22 (1967), 77 - 102).

[Kat-St 2] A. Katok, A. Stepin, Metric properties of homeomorphisms that preserve measure, Usp. Mat. Nauk, 25(2) (1970), 193 - 220 (Engl. Transl. 'Russian Math. Surveys', 25 (1970), $191-220)$.

[Kea] M. Keane, Contractibility of the automorphism group of a nonatomic measure space, Proc. Amer. Math. Soc., 26 (1970), 420 - 421.

[Ke-Mil] A. Kechris, B. Miller, Topics in Orbit Equivalence Theory, Lect. Notes in Math., v. 1852, 2004.

[Ke-Ros] A. Kechris, C. Rosendal, Turbulence, amalgamation and generic automorphisms of homogenuous structures, ArXiv:math.LO/0409567 v2 30 September 2004.

[Ki] J. King, The commutant is the weak closure of the powers, for rank-1 transformations, Erg. Th. Dynam. Syst., 6 (1986), 363-384.

[Kre] U. Krengel, Entropy of conservative transformations, Z. Wahrscheinlichkeitstheorie und Verw. Gebiete, 7 (1966), 161 - 181.

[Ma] H. Matui, Topological orbit equivalence of locally compact Cantor minimal systems, Erg. Th. Dyn. Syst., 22 (2002), $1971-1903$.

[M] K. Medynets, Cantor aperiodic systems and Bratteli diagrams, preprint, 2004.

[Mer] R. Mercer, The full group of a countable measurable equivalence relation, Proc. Amer. Math. Soc., 117 (1993), $323-333$.

[N] M. Nadkarni, Basic Ergodic Theory, 2nd Edition, Birkhäuser, 1998. 
[O 1] J. Oxtoby, Approximation by measure-preserving homeomorphisms, Recent Advances in Topological Dynamics, Lecture Notes in Mathematics, 318 (1973), 206 -217.

[O 2] J. Oxtoby, Measure and category. A survey of the analogies between topological and measure spaces. Second edition. Graduate Texts in Mathematics, 2. Springer-Verlag, New York-Berlin, 1980. $\mathrm{x}+106 \mathrm{pp}$

[O-U] J. Oxtoby, S.M. Ulam, measure-preserving homeomorphisms and metrical transitivity, Ann. of Math., 42 (1941), 874-920.

[Pe] K. Petersen, Ergodic Theory. Cambridge University Press, 1983.

[Pr 1] V.S. Prasad, Generating dense subgroups of measure preserving transformations, Proc. Amer. Math. Soc., 83 (1981), $286-288$.

[Ro 1] V.A. Rokhlin, Selected topics from the metric theory of dynamical systems (Russian), Usp. Mat. Nauk (N.S.), 4 (1949), no. 2, 57-128 (Engl. Transl. in 'Amer. Math. Soc. Translations', 49 (1966), $171-240)$.

[Ro 2] V.A. Rokhlin, A 'general' measure preserving transformation is not mixing, Dokl. Akad. Nauk SSSR, 60 (1948), 349 - 351.

[Ro 3] V.A. Rokhlin, Entropy of metric endomorphisms (Russian), Dokl. Akad. Nauk SSSR, 124 (1959), $980-983$.

[Sa] U. Sachdeva, On category of mixing in infinite measure spaces, Math. Systems Theory, 5 (1971), $319-330$.

[Sea] M. Sears, Topologies on the group of self homeomorphisms of the Cantor set, Mat. Časopis Sloven. Akad. Vied, 21 (1971), 227-232.

[Sh] R.M. Shortt, Normal subgroups of measurable automorphisms, Fund. Math., 135 (1990), $177-187$.

[W] B. Weiss, Measurable dynamics, Contemporary Mathematics, 26 (1984), 395 - 421.

Department of Mathematics, Institute for Low Temperature Physics, 47 Lenin ave., 61103 KhARKOV, UKRAINE

E-mail address: bezuglyi@ilt.kharkov.ua

Faculty of Mathematics and Computer Sciences, Nicolas Copernicus University, ul. Chopina 12/18, 87 - 100 Torun, Poland

E-mail address: jkwiat@mat.uni.torun.pl

Department of Mathematics, Institute for Low Temperature Physics, 47 Lenin ave., 61103 KhaRKov, UKRAINe

E-mail address: medynets@ilt.kharkov.ua 